ARTICLE

\title{
Fibrocyte-like cells mediate acquired resistance to anti-angiogenic therapy with bevacizumab
}

\author{
Atsushi Mitsuhashi ${ }^{1, \star}$, Hisatsugu Goto ${ }^{1, \star}$, Atsuro Saijo ${ }^{1}$, Van The Trung${ }^{1}$, Yoshinori Aono ${ }^{1}$, Hirokazu Ogino ${ }^{1}$ \\ Takuya Kuramoto', Sho Tabata1, Hisanori Uehara², Keisuke Izumi ${ }^{2}$, Mitsuteru Yoshida ${ }^{3}$, Hiroaki Kobayashi ${ }^{4}$, \\ Hidefusa Takahashi ${ }^{5}$, Masashi Gotoh ${ }^{6}$, Soji Kakiuchi ${ }^{1}$, Masaki Hanibuchi ${ }^{1}$, Seiji Yano ${ }^{7}$, Hiroyasu Yokomise ${ }^{6}$, \\ Shoji Sakiyama ${ }^{3} \&$ Yasuhiko Nishioka ${ }^{1,8}$
}

Bevacizumab exerts anti-angiogenic effects in cancer patients by inhibiting vascular endothelial growth factor (VEGF). However, its use is still limited due to the development of resistance to the treatment. Such resistance can be regulated by various factors, although the underlying mechanisms remain incompletely understood. Here we show that bone marrow-derived fibrocyte-like cells, defined as alpha-1 type I collagen-positive and CXCR4-positive cells, contribute to the acquired resistance to bevacizumab. In mouse models of malignant pleural mesothelioma and lung cancer, fibrocyte-like cells mediate the resistance to bevacizumab as the main producer of fibroblast growth factor 2. In clinical specimens of lung cancer, the number of fibrocyte-like cells is significantly increased in bevacizumabtreated tumours, and correlates with the number of treatment cycles, as well as CD31-positive vessels. Our results identify fibrocyte-like cells as a promising cell biomarker and a potential therapeutic target to overcome resistance to anti-VEGF therapy.

\footnotetext{
${ }^{1}$ Department of Respiratory Medicine and Rheumatology, Institute of Biomedical Sciences, Tokushima University Graduate School, 3-18-15 Kuramoto-cho, Tokushima, Tokushima 770-8503, Japan. ${ }^{2}$ Department of Molecular and Environmental Pathology, Institute of Biomedical Sciences, Tokushima University Graduate School, 3-18-15 Kuramoto-cho, Tokushima, Tokushima 770-8503, Japan. ${ }^{3}$ Department of Thoracic, Endocrine Surgery and Oncology, Institute of Biomedical Sciences, Tokushima University Graduate School, 3-18-15 Kuramoto-cho, Tokushima, Tokushima 770-8503, Japan. ${ }^{4}$ Department of Thoracic Surgery, Fukui-ken Saiseikai Hospital, Funabashi 7-1, Wadanaka-cho, Fukui, Fukui 918-8503, Japan. ${ }^{5}$ Department of Internal Medicine, Municipal Tsuruga Hospital, 1-6-60 Mishima-cho, Tsuruga, Fukui 914-8502, Japan. ${ }^{6}$ Department of General Thoracic, Breast and Endocrinological Surgery, Faculty of Medicine, Kagawa University, 1750-1 Ikenobe, Miki-cho, Kita-gun, Kagawa 761-0793, Japan. ${ }^{7}$ Division of Medical Oncology, Cancer Research Institute, Kanazawa University, 13-1 Takara-machi, Kanazawa, Ishikawa 920-0934, Japan. ${ }^{8}$ JST, CREST, Tokyo 102-0076, Japan. * These authors contributed equally to this work. Correspondence and requests for materials should be addressed to Y.N. (email: yasuhiko@tokushima-u.ac.jp).
} 
$\mathrm{n}$ adequate blood supply is essential for cancer cells to survive and grow, thus, the concept of inhibiting tumour angiogenesis has been applied to cancer therapy ${ }^{1,2}$. Bevacizumab is a monoclonal antibody which blocks vascular endothelial growth factor (VEGF) that is the most potent proangiogenic factor to mediate multiple steps of tumour angiogenesis ${ }^{3,4}$. The results from phase III clinical trials have demonstrated that the addition of bevacizumab to conventional chemotherapy improves the response rate and prolongs survival of patients with non-small cell lung cancer (NSCLC) and colon cancer ${ }^{5,6}$. However, in 2011, an announcement was made by the US Food and Drug Administration revoking the approval of bevacizumab for the treatment of metastatic breast cancer because of its insufficient efficacy and safety ${ }^{7}$. The possible reasons for the disappointing clinical results may include the lack of biomarkers for the efficacy of or resistance to bevacizumab treatment. A significant number of patients either do not respond to anti-VEGF agents or develop resistance to them after an initial response ${ }^{8,9}$. Therefore, it is crucial to investigate the mechanism(s) of resistance and to identify biomarkers for intrinsic and/or acquired resistance to bevacizumab treatment to develop more effective cancer therapies.

For the mechanism of the resistance to anti-VEGF therapy, the induction of hypoxia inducible factor (HIF) in tumour cells seems to be the most intensively reported. The upregulated expression of HIF in tumour cells under the hypoxic conditions initiated by the inhibition of angiogenesis induces various pro-angiogenic factors to regenerate microvessels in the tumour $2,8,10,11$. For host cell-mediated resistance, the involvement of tumour-associated macrophages (TAM), myeloid-derived suppressor cells (MDSC) and vascular pericytes has been reported in mice ${ }^{12-16}$. Taken together, the resistance to anti-VEGF therapy is regulated by diverse mechanisms, including those related to the tumour and host cells, although their respective functions remain incompletely understood. Moreover, the current knowledge in this field is mainly based on the observations in mouse models. Verifying the major mechanism(s) of resistance in human tumours is crucial.

In this study, we hypothesize that there are still uncovered molecular and/or cellular mechanisms that regulate the resistance to bevacizumab. To assess this hypothesis, we use mouse models of malignant pleural mesothelioma (MPM) and lung cancer, and lung cancer clinical specimens resected from patients after bevacizumab therapy to explore the mechanism of resistance to bevacizumab. We identify bone marrow-derived fibrocyte-like cells, which are double-positive for alpha-1 type I collagen and CXCR4, as a previously unrecognized cell type involved in the acquired resistance to bevacizumab via their production of fibroblast growth factor 2 (FGF2). Given that the soluble factors have not been successfully developed as a practical biomarker for the resistance to bevacizumab in clinic, fibrocytelike cells may be a promising cell biomarker and a potential therapeutic target to overcome resistance to anti-VEGF therapy.

\section{Results}

Acquired resistance to bevacizumab in mouse models. Initially, to investigate the mechanism by which tumours develop resistance to VEGF inhibition, we orthotopically or intravenously injected immunodeficient mice with human MPM cell lines (Y-MESO-14 and EHMES-10 cells) or human lung adenocarcinoma cell lines (PC14PE6 and A549 cells) that highly express VEGF ${ }^{17-20}$. Orthotopically injected Y-MESO-14 and EHMES-10 cells produced thoracic tumours and pleural effusion, and the intravenously injected PC14PE6 cells and A549 cells produced multiple lung metastatic colonies. PC14PE6 cells also produced pleural effusion. Seven days after tumour injection, continuous treatment with bevacizumab was started. As expected, bevacizumab treatment prolonged the survival of mice injected with any of these four cell lines compared with the control group (Fig. 1a) (Y-MESO-14; $P=0.0355$, EHMES-10; $P=0.0046$, PC14PE6; $P=0.0002$, A549; $P=0.0780$, by a log-rank test). In the models of Y-MESO-14 and PC14PE6 cells we confirmed that bevacizumab effectively suppressed the tumour growth at day 28 and 35 after tumour cell inoculation, respectively (Fig. $1 \mathrm{~b}$ and Table 1). Interestingly, the mice became moribund because of tumour progression despite continuous bevacizumab treatment (Fig. 1a,b), suggesting that the tumours exhibited resistance to bevacizumab. To investigate whether this resistance to bevacizumab was intrinsic or acquired, tumours were harvested at different time points, and the microvessel density (MVD) was immunohistochemically evaluated. MVD was significantly reduced by bevacizumab treatment; however, it gradually increase over time with continuous bevacizumab treatment (Fig. 1c,d and Supplementary Fig. 1), suggesting that the resistance to bevacizumab in these tumours was acquired. The subsequent experiments were performed using MPM cell lines, or otherwise indicated.

Induction of stromal FGF2 in bevacizumab-resistant tumours. To identify the molecule(s) that contributes to the acquired resistance to anti-VEGF therapy, we engineered human- or mouse-specific primers to determine the differential changes in the tumour (human) and/or stromal (mouse) gene expression by performing a quantitative PCR (qPCR) analysis. The expression of tumour cell-produced pro-angiogenic ligands was examined using human-specific primers on day 28 after tumour cell (Y-MESO-14) inoculation, and the upregulation of VEGFA and FGF1 was observed. However, the expression of these molecules was not changed or was under the limit of detection when the protein expression was evaluated (Supplementary Fig. 2). On the other hand, a qPCR analysis using mouse-specific primers revealed that the expression of VEGFA, FGF2, FGFR2 and platelet-derived growth factor receptor A (PDGFRA) was upregulated in the bevacizumab-treated tumour stroma (Fig. 2a). As both the ligand and receptor for stromal FGF signalling were increased in the bevacizumab-treated tumours, we focused on stromal FGF2 as a candidate molecule for the acquired resistance to anti-VEGF therapy, and confirmed that the protein expression of mouse FGF2 was significantly increased in the pleural effusion of bevacizumab-treated Y-MESO-14 tumour-bearing mice (Fig. 2b). The protein level of mouse VEGF-A was also increased by 1.4 -fold in the pleural effusion of bevacizumab-treated mice compared with the control mice, although it did not reach to the significance (Fig. 2c). Furthermore, an immunohistochemical analysis demonstrated that the number of FGF2-positive cells to significantly increase in bevacizumab-treated tumours compared with the control-treated tumours on day 28 after tumour cell inoculation (Fig. 2d,e).

FGFR inhibitor improves bevacizumab resistance. To verify the role of FGF2 in bevacizumab resistance, tumour (Y-MESO-14 cells)-bearing mice were treated with a FGFR inhibitor (BGJ-398 $)^{21}$ in combination with bevacizumab. The combination therapy further prolonged the survival of mice compared with the treatment with bevacizumab or BGJ-398 alone (Fig. 2f) (control vs bevacizumab; $P=0.0042$, control vs BGJ-398; $P=0.0017$, bevacizumab vs combination; $P=0.005$, BGJ-398 vs combination; $P=0.0078$ by a log-rank test). In addition, MVD was significantly reduced by the combination treatment (Fig. 2g,h). The combination treatment of FGF2-neutralizing Ab 

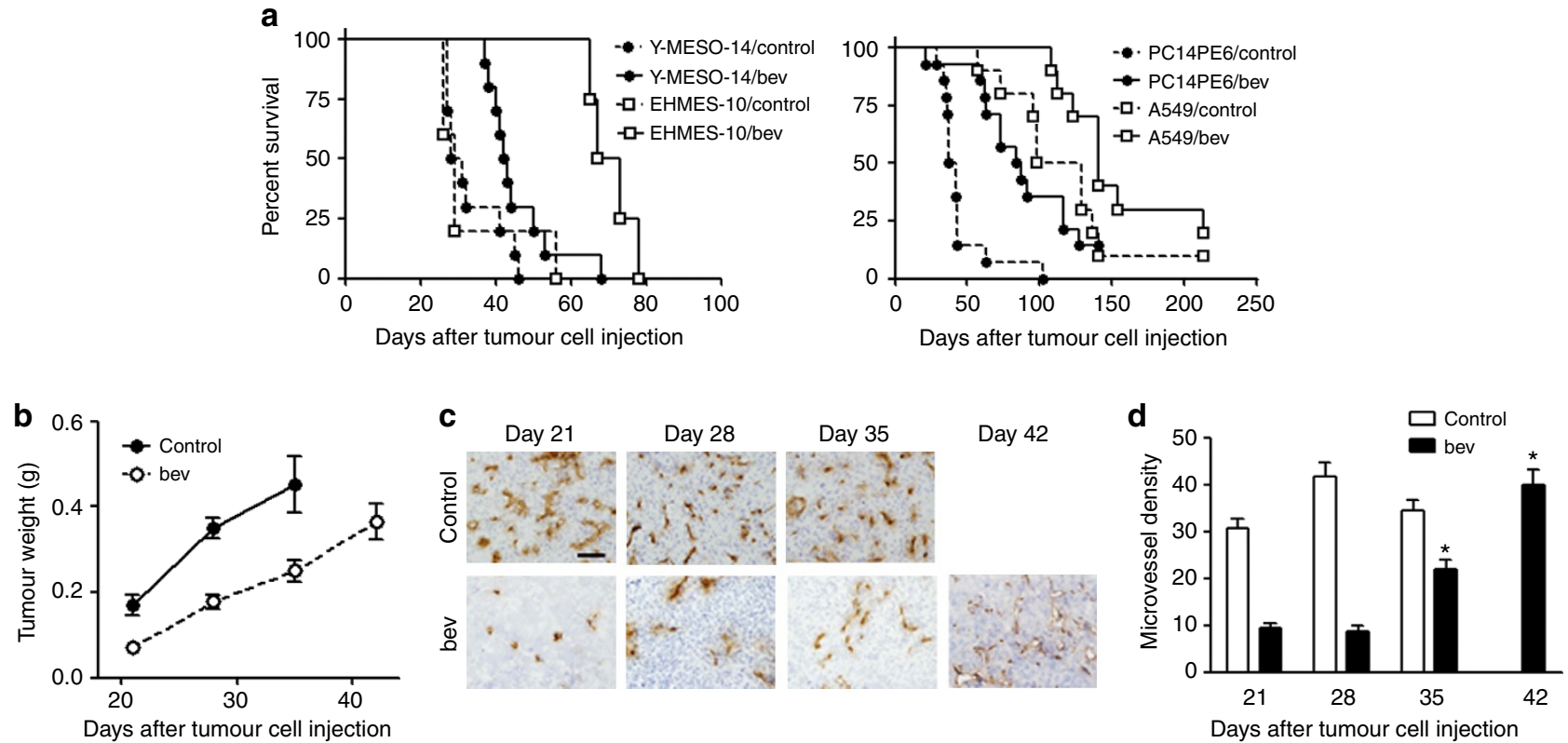

Figure 1 | Continuous treatment with bevacizumab induces acquired resistance in vivo. (a) The survival curve of tumour-bearing mice treated with bevacizumab (bev) beginning from seven days after tumour cell injection. (left) Human MPM cell lines (Y-MESO-14 and EHMES-10) were injected orthotopically. (right) Human lung cancer cell lines (PC14PE6 and A549) were injected via the tail vein. (b) The weights of Y-MESO-14 intrathoracic tumours from the control and bevacizumab (bev)-treated groups at different time points. (c) Representative images of sections from Y-MESO-14 intrathoracic tumours stained for CD31. The tumours were harvested at different time points from the control and bevacizumab (bev)-treated groups. Scale bar, $200 \mu \mathrm{m}$. (d) Quantitative evaluation of MVD (quantified as the total number of $\mathrm{CD} 31^{+}$structures/HPF) in each tumour of mice ( $n=5$ per group) studied in $\mathbf{c}$. The data are shown as the means \pm s.e.m ${ }^{\star} P<0.05$ by a one-way ANOVA.

\section{Table 1 | Effects of bevacizumab on thoracic tumours in mice.}

\section{Y-MESO-14}

Control $(n=4)$

Bev $10 \mu g(n=5)$

\begin{tabular}{cc}
\multicolumn{2}{c}{ Thoracic tumour } \\
\hline Incidence & Weight $(\mathbf{m g})$ \\
$4 / 4$ & $260(210-330)$ \\
$5 / 5$ & $170(100-250)^{\star}$
\end{tabular}

\begin{tabular}{cc} 
& Pleural effusion \\
\hline Incidence & Volume $(\boldsymbol{\mu l})$ \\
$4 / 4$ & $290(200-700)$ \\
$4 / 5$ & $50(0-250)^{\star}$
\end{tabular}

\section{PC14PE6}

Control $(n=7)$

Control $(n=7)$
Bev $10 \mu g(n=7)$

Bev $30 \mu g(n=7)$

\begin{tabular}{ccc} 
& \multicolumn{2}{l}{ Lung } \\
\cline { 2 - 3 } Weight (mg) & \multicolumn{3}{c}{ Metastasis } \\
\cline { 2 - 3 } & Incidence & Number \\
\hline $480(320-880)$ & $7 / 7$ & $62(25-87)$ \\
$180(170-200)^{\dagger}$ & $6 / 7$ & $17(0-42)^{\dagger}$ \\
$180(150-250)^{\dagger}$ & $7 / 7$ & $29(4-101)^{\star}$
\end{tabular}

$-$

Incider

Pleural effusion

MPM, malignant pleural mesothelioma; SCID, severe combined immunodeficient

Y-MESO-14 (human MPM) cells were orthotopically injected to SCID mice, and PC14PE6 (human lung adenocarcinoma) cells were intravenously injected to nude mice. These mice were treated with bevacizumab (Bev) from day 7 after the tumour cell injection, and thoracic tumour, lung metastasis and pleural effusion were evaluated on day 28 (Y-MESO-14) or on day 35 (PC14PE6) after tumour cell injection. Values are the median (minimum-maximum).

*Statistically significant difference compared with control group $(P<0.05)$ by the Mann-Whitney-U-test

$\dagger$ Statistically significant difference compared with control group $(P<0.01)$ by the Mann-Whitney-U-test.

and bevacizumab also reduced MVD in the tumour produced by Y-MESO-14 cells compared with the bevacizumab treatment in combination with control IgG (Fig. 2i,j). These results indicated that stromal FGF2 plays a role in the resistance to anti-VEGF therapy.

Fibrocyte-like cells as FGF2-expressing cells in the tumour. We next examined what type of cells expressed FGF2 in the bevacizumab-resistant tumour environment. To identify the responsible cell type(s), we immunohistochemically assessed various cell markers (Fig. 3a and Supplementary Fig. 3a). FGF2 expression did not co-localize with CD68, F4/80 or Gr-1, suggesting that FGF2 was not expressed by macrophages or MDSCs. However, many cells did co-express FGF2 and collagen type I or CXCR4. Interestingly, collagen type I and CXCR4 were predominantly co-localized, and the number of double-positive cells was significantly increased in the bevacizumab-resistant tumours (Fig. 3b,c and Supplementary Fig. 3b,c).

The unique phenotype with the co-expression of collagen type I and CXCR4 was compatible to that of fibrocytes, which express hematopoietic progenitor cell markers (CD45, CD34) and produce extracellular matrix proteins, and also express chemokines such as CXCR4 (ref. 22). An additional immunohistochemical analysis showed that some collagen type I-positive cells also expressed CD45 (Fig. 3d). Furthermore, these collagen 

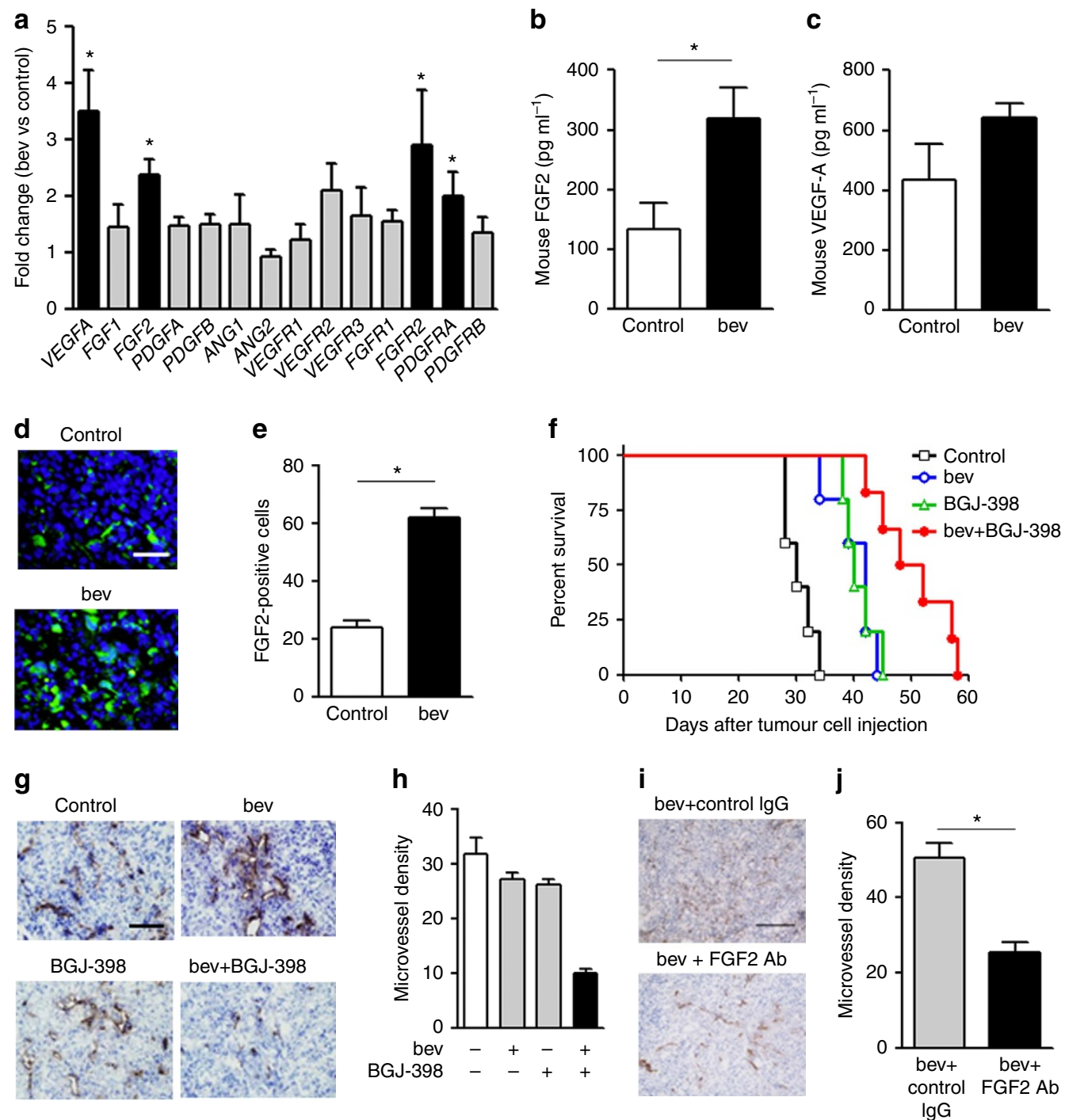

Figure 2 | Host-derived FGF2 is upregulated in the intrathoracic Y-MESO-14 tumours resistant to bevacizumab treatment. (a) A comparison of mouse mRNA expression of pro-angiogenic factors in the intrathoracic Y-MESO-14 tumours treated with or without bevacizumab (bev). The fold-changes in mRNA expression of the bevacizumab-treated tumours compared with control tumours are shown. ${ }^{\star} P<0.05$ by the Mann-Whitney- $U$ test. The changes in the amounts of mouse (b) FGF2 ( $n=8$ per group) and (c) VEGF ( $n=6$ per group) in the pleural effusion of tumour (Y-MESO-14)-bearing mice treated with or without bevacizumab (bev). ${ }^{\star} P<0.05$ by the Mann-Whitney-U-test. (d) Representative images of FGF2-positive cells (green) in the sections from Y-MESO-14 intrathoracic tumours treated with or without bevacizumab (bev). Scale bar, $100 \mu \mathrm{m}$. (e) The number of FGF2-positive cells in the Y-MESO-14 tumours treated with bevacizumab (bev) compared with the control group ( $n=9$ per group). ${ }^{\star} P<0.01$ by the Mann-Whitney- $U$-test. (f) The survival curves of the tumour (Y-MESO-14)-bearing mice treated with bevacizumab (bev), BGJ-398 (an FGFR inhibitor), or both. Bevacizumab was continuously given from day 7 after tumour cell injection. BGJ-398 was given from days 14-28. (g) Representative images of the CD31-positive vessels in the tumours harvested from each group. Scale bar, $200 \mu \mathrm{m}$. (h) Evaluation of microvessel density (MVD, quantified as the total number of CD31 ${ }^{+}$structures/HPF) in the tumours from each group ( $n=12$ per group). ${ }^{\star} P<0.01$ by a one-way ANOVA. (i) Representative images of the CD31-positive vessels in the tumours harvested from mice treated with bevacizumab (bev) in combination with FGF2 Ab or control lgG. Scale bar, $200 \mu$ m. (j) Evaluation of MVD in each group ( $n=12$ per group). ${ }^{\star} P<0.01$ by the Mann-Whitney-U-test. All data are shown as the means \pm s.e.m.

type $\mathrm{I}^{+} / \mathrm{CXCR} 4^{+}$cells were confirmed to produce $\mathrm{FGF} 2$ (Fig. 3e), suggesting that the collagen type $\mathrm{I}^{+} /$CXCR4 ${ }^{+}$ fibrocyte-like cells play an essential role in the bevacizumab resistance of tumours by expressing FGF2. There was still a possibility that the collagen type $\mathrm{I}^{+}$cells were macrophages, because macrophages may produce low levels of collagen, in addition to CXCR4 (ref. 23). However, neither CD68 nor F4/80 co-localized with collagen type I (Fig. 3f).

To further confirm the characteristics of the collagen type $\mathrm{I}^{+} / \mathrm{CXCR} 4{ }^{+}$fibrocyte-like cells, we sought the origin of these cells. Mice were given a bone marrow transplant (BMT) from GFP transgenic mice before cancer cell inoculation, and the collagen type I-positive cells in the tumour were assessed to determine whether they were GFP-positive. Interestingly, the number of GFP-positive cells was significantly increased in the bevacizumab-resistant tumours (Fig. 4a,b), and the collagen type $\mathrm{I}^{+} / \mathrm{CXCR} 4{ }^{+}$cells were also GFP-positive (Fig. 4c), suggesting that the origin of the collagen type $\mathrm{I}^{+} / \mathrm{CXCR} 4^{+}$cells was the bone marrow. These results collectively indicated that the collagen type $\mathrm{I}^{+} / \mathrm{CXCR} 4^{+}$cells in the tumour have the distinctive features of fibrocytes. In addition, it was also confirmed that FGF2 was predominantly produced by GFP-positive cells in bevacizumab-resistant tumour in bone marrow chimeric mice (Fig. 4d). 
a FGF2

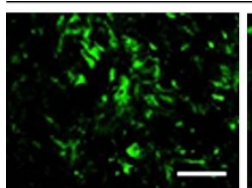

CD68
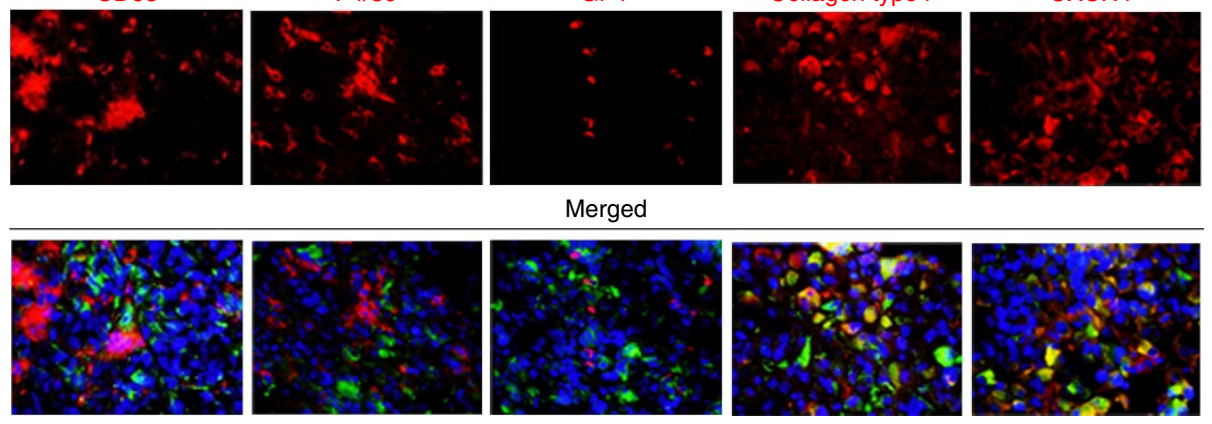

b
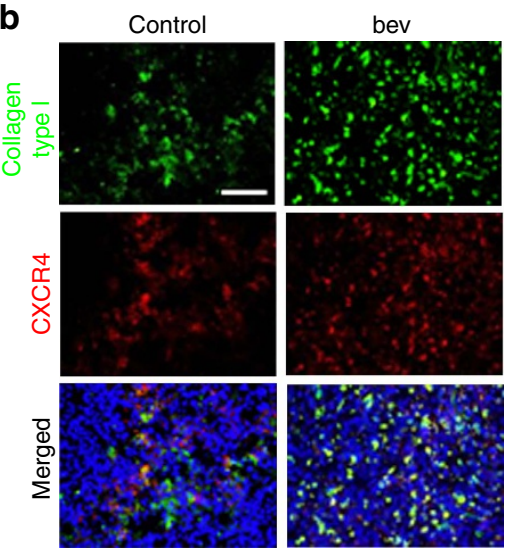

C
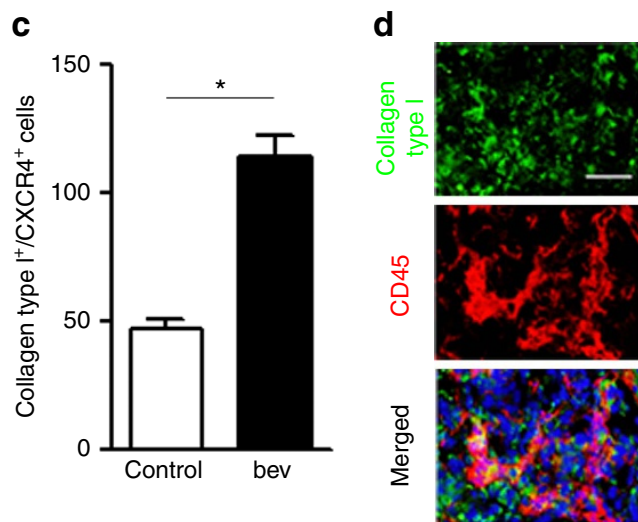

e

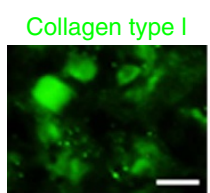

FGF2
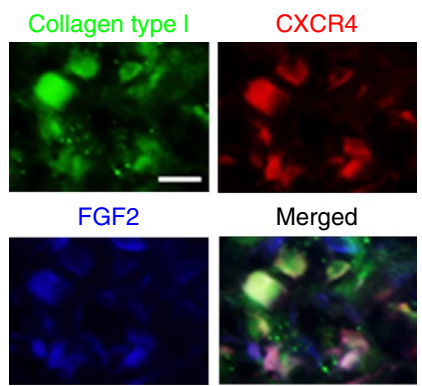

f Collagen type |

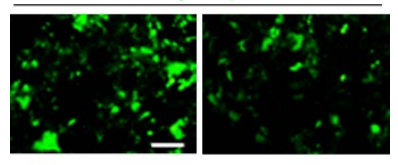

CD68
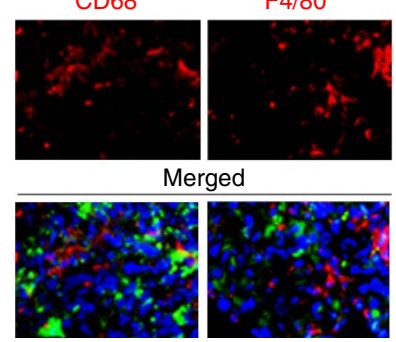

Figure 3 | The identification of collagen type $\mathbf{I}^{+}$/CXCR4 ${ }^{+}$cells as FGF2-producing cells in the intrathoracic tumours produced by Y-MESO-14 cells. (a) Representative images of tumour sections from mice treated with bevacizumab. FGF2 (green) was co-stained along with CD68, F4/80, Gr-1, collagen type I or CXCR4 (red). Scale bar, $100 \mu \mathrm{m}$. (b) Double staining of collagen type I and CXCR4 in tumours from mice treated with or without bevacizumab (bev). Scale bar, $200 \mu \mathrm{m}$. (c) The number of double-positive cells in the tumours was compared between the control- and bevacizumab (bev)-treated groups ( $n=20$ per group). The data are shown as the means \pm s.e.m. ${ }^{*} P<0.01$ by the Mann-Whitney- $U$-test. (d) Representative images of double staining for collagen type I and CD45 in Y-MESO-14 tumours treated with bevacizumab. Scale bar, $100 \mu \mathrm{m}$. (e) Representative images of triple staining for collagen type I, CXCR4 and FGF2 in the tumours treated with bevacizumab. Scale bar, $50 \mu \mathrm{m}$. (f) Representative images of double staining for collagen type I (green) and CD68 or F4/80 (red) in Y-MESO-14 tumours treated with bevacizumab. Scale bar, $100 \mu \mathrm{m}$.

We finally performed the flow cytometric analysis to confirm that the number of fibrocyte-like cells was increased in the bevacizumab-resistant tumour. Single-cell suspension of controlor bevacizumab-treated tumour was stained with $\mathrm{CD} 45$, collagen type I and CXCR4 to detect fibrocyte-like cells. The percentage of CD45 $5^{+} /$collagen type $\mathrm{I}^{+} / \mathrm{CXCR} 4^{+}$cells was significantly increased in the bevacizumab-resistant tumour (Fig. 5a,b). The average number of leukocytes $\left(\mathrm{CD} 45^{+}\right.$cells) in control- and 

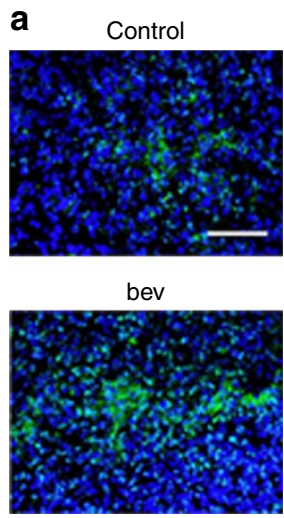

b
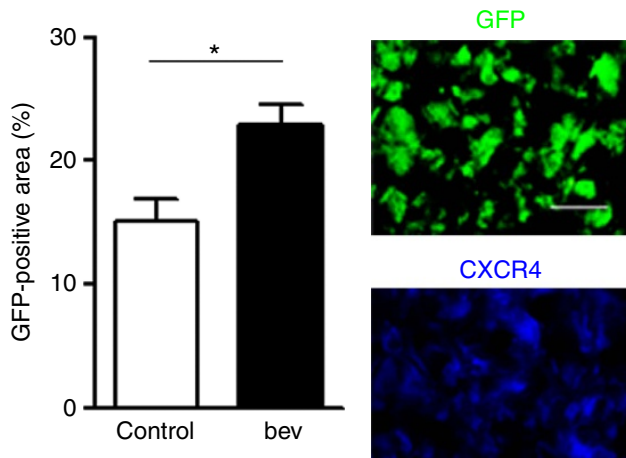

CXCR4
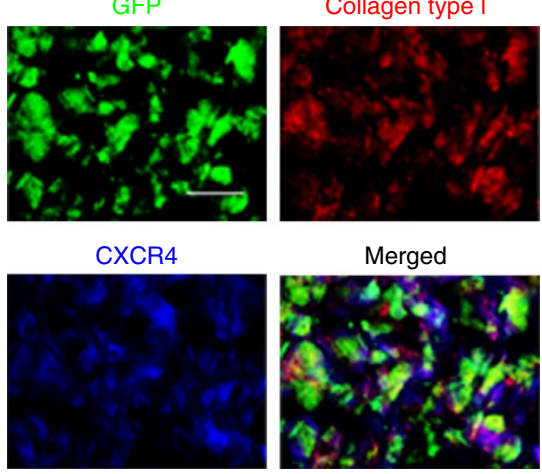

Merged

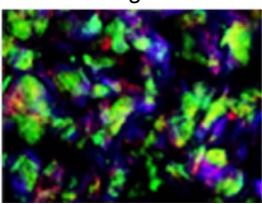

d
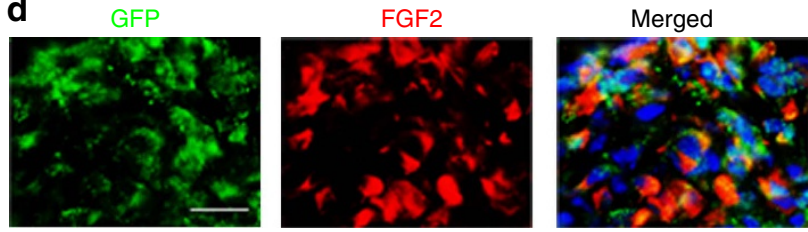

Figure 4 | The recruitment of bone marrow-derived cells in bevacizumab-resistant tumour. Mice were given a bone marrow transplant (BMT) from GFP transgenic mice before cancer cell inoculation. (a) Representative images of GFP-positive bone marrow cells (green) recruited into the control or bevacizumab (bev)-treated tumours. Scale bar, $200 \mu \mathrm{m}$. (b) The percentages of GFP-positive areas in the tumours were compared between the controland bevacizumab (bev)-treated groups ( $n=8$ per group). The data are shown as the means \pm s.e.m. ${ }^{\star} P<0.01$ by the Mann-Whitney-U-test. (c) Representative images of triple staining for GFP, collagen type I and CXCR4 in the tumours treated with bevacizumab. Scale bar, $50 \mu \mathrm{m}$. (d) Representative images of double staining for GFP and FGF2 in the tumours treated with bevacizumab. Scale bar, $100 \mu \mathrm{m}$.

bevacizumab-treated tumour was $1.56 \times 10^{4}$ and $2.19 \times 10^{4}$ cells per $1 \mathrm{mg}$ of tumour tissue, respectively (not significant with Student's $t$-test), whereas the number of fibrocyte-like cells was $1.12 \times 10^{3}$ and $3.80 \times 10^{3}$ per $1 \mathrm{mg}$ of tumour tissue, respectively $(P<0.05$ with Student's $t$-test $)$. On the other hand, the percentage of $\mathrm{CD} 45^{+} / \mathrm{Gr}-1^{+}$MDSCs was not changed by bevacizumab treatment (Fig. $5 \mathrm{c}$ ), whereas the percentage of CD45 ${ }^{+} / \mathrm{F} 4 / 80^{+}$ macrophages was slightly increased with bevacizumab treatment (Fig. 5c).

Since the limitation of this preclinical study is that we used immunodeficient mice that lack lymphocytes, we also employed syngeneic mouse model using B16 melanoma cells and C57BL/6 mice to verify the role of other immune cells in the acquired resistance to anti-VEGF therapy (Supplementary Fig. 4). We subcutaneously injected B16 cells to C57BL/6 mice, and treated these mice with SU5416, a VEGF receptor inhibitor, continuously from day 5 after tumour cell inoculation. Similar to the results obtained from immunodeficient mice experiment, the tumour grew and MVD was increased regardless of SU5416 treatment after the initial response (Supplementary Fig. $4 \mathrm{a}-\mathrm{c}$ ). These tumour regrowth were suppressed by combination treatment with SU5416 and BGJ-398 (Supplementary Fig. 4d). In this model, the fibrocyte-like cells were detected as $\mathrm{CD} 45^{+} / \mathrm{FSP}-1^{+}$cells, and the increased number of tumour-infiltrated CD $45^{+} / \mathrm{FSP}-1^{+}$cells after SU5416 treatment were confirmed by immunofluorescence (Supplementary Fig. 4e,f). The effect of SU5416 treatment on the infiltration of lymphocytes into the tumour was also examined by immunofluorescence. The numbers of tumour-infiltrated CD3- or CD19-positive cells were significantly low compared with that of fibrocyte-like cells, and did not change by SU5416 treatment (Supplementary Fig. 4g).

Character of fibrocyte-like cells in the resistant tumour. To confirm the results from immuonofluorescence and flow cytometry indicating that the number of fibrocyte-like cells was increased and that these were the FGF2-producing cells in the bevacizumab-resistant tumours, we isolated and characterized the fibrocyte-like cells from mouse tumour tissue. Before this, fibrocyte-like cells and fibroblasts were differently isolated from mouse normal lungs, and their gene expression profiles were compared. As reported previously, fibrocyte-like cells expressed higher levels of CXCR4 and CD45, and relatively lower levels of COL1A1 compared with fibroblasts (Fig. 6a) ${ }^{22}$. FGF2 expression in fibrocyte-like cells was lower than that in fibroblasts. When fibrocyte-like cells were isolated from the mouse tumour tissues, the number of these cells was significantly increased in the bevacizumab-resistant tumours compared with the controltreated tumours (Fig. 6b). On the other hand, the expression of FGF2, as well as CXCR4, CD45 and COL1A1, in the isolated fibrocyte-like cells was similar between the tumours (Fig. 6c), suggesting that the increase of FGF2 in bevacizumab-treated tumours was due to the increase in the number of these cells.

Mechanism in the recruitment of fibrocyte-like cells. To investigate the mechanism(s) underlying the functions and recruitment of fibrocyte-like cells in the bevacizumab-resistant tumours, in vitro cell-proliferation experiments were performed using human fibrocyte-like cells derived from peripheral blood mononuclear cells. We first confirmed that bevacizumab or BGJ-398 inhibited VEGF- or FGF2-induced proliferation of human umbilical vein endothelial cells (HUVECs), respectively (Supplementary Fig. 5). As reported previously, the supernatant of fibrocyte-like cells induced the proliferation of the HUVECs ${ }^{24}$, but interestingly, their proliferation was inhibited only by BGJ-398, not by bevacizumab (Supplementary Fig. 5), supporting our results showing that the fibrocyte-like cells express FGF2.

To determine the mechanism by which fibrocyte-like cells were recruited into the tumour environment, we performed humanspecific gene profiling using tumour tissues (Y-MESO-14). Among the genes that were relatively upregulated in the 
a

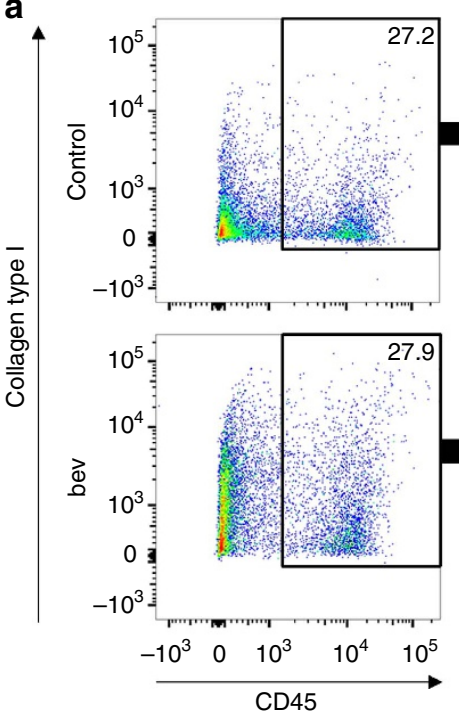

C

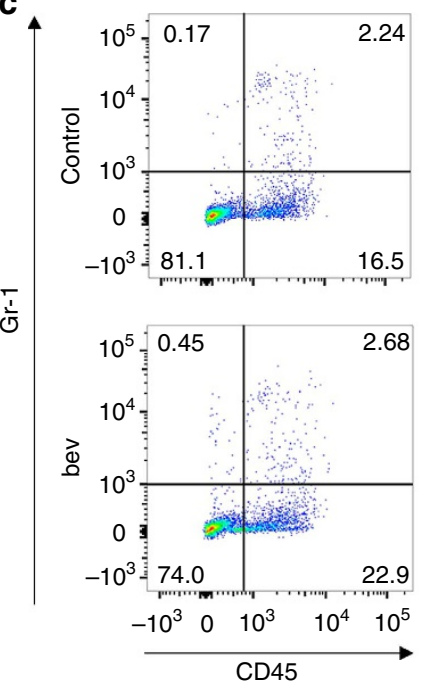

b

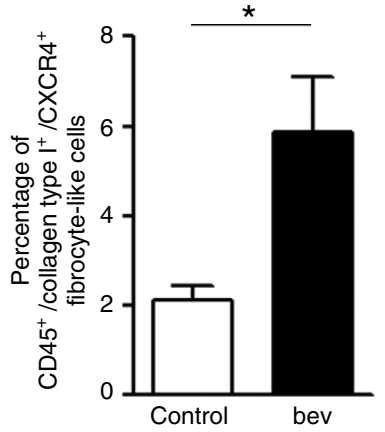

Figure 5 | The isolation of mouse fibrocyte-like cells from Y-MESO-14 tumours by flow cytometry. (a) Representative analysis of the detection of fibrocyte-like cells. The tumour tissues were harvested and single-cell suspended from control- or bevacizumab (bev)-treated mice on day 28. Fibrocytelike cells were detected as $\mathrm{CD} 45^{+} /$collagen type $\mathrm{I}^{+} / \mathrm{CXCR} 4^{+}$population. Each samples contained tumour tissues from two mice. (b) The comparison of the percentage of $\mathrm{CD}_{4} 5^{+} /$collagen type $\mathrm{I}^{+} / \mathrm{CXCR} 4^{+}$cells between control- and bevacizumab (bev)-treated group ( $n=3$ in each group, each samples contained tumour tissues from two mice). ${ }^{\star} P<0.05$ by Student's $t$-test. The data are shown as the means \pm s.e.m. (c) MDSCs or Macrophages were detected as $\mathrm{CD} 45^{+} / \mathrm{Gr}-1^{+}$cells or $\mathrm{CD} 45^{+} / \mathrm{F} 4 / 80^{+}$cells, respectively. Each samples contained tumour tissues from two mice. Data are the representative of two experiments with similar results.

bevacizumab-resistant tumours compared with the control tumours, we focused on CXCL12, the ligand of CXCR4 (Supplementary Fig. 6). Because CXCL12 is the target molecule of HIF- $1 \alpha$, the hypoxic condition resulting from bevacizumab treatment could lead the tumour cells to produce CXCL12, which would in turn prompt the CXCR4 ${ }^{+}$cells to migrate into the tumour. Indeed, the area of hypoxia was significantly increased (Fig. 7a) and the CXCL12 expression was confirmed to be significantly upregulated in the bevacizumab-treated Y-MESO-14 tumours compared with the control tumours (Fig. 7b, left panel). CXCL12 expression was also induced in a chemical hypoxic condition induced by treating Y-MESO-14 cells with deferoxamine (DFX) in vitro (Fig. 7b, right panel).

We then examined whether the CXCL12 induced by the hypoxic condition might be able to regulate the migration of fibrocyte-like cells. As expected, the number of migrated fibrocyte-like cells was strongly increased when the cells were treated with the supernatant of Y-MESO-14 cells cultured under hypoxic conditions. Moreover, this induction of migration was inhibited by a CXCR4 inhibitor (AMD3100) ${ }^{25}$, indicating the important role of the CXCL12-CXCR4 axis in the migration of fibrocyte-like cells (Fig. 7c,d).

To further verify the importance of the fibrocyte-like cells recruited by the CXCL12-CXCR4 axis in the development of bevacizumab resistance in vivo, tumour (Y-MESO-14)-bearing mice were treated with bevacizumab and/or AMD3100. The combination of AMD3100 with bevacizumab further inhibited tumour growth (Fig. 7e), angiogenesis (Fig. 7f) and fibrocyte-like cells recruitment (Fig. 7g), suggesting that CXCR4 inhibition could partially overcome the development of bevacizumab resistance by inhibiting the re-angiogenesis mediated by fibrocyte-like cells. On the other hand, AMD3100 is known to induce the mobilization of hematopoietic stem cells (HSCs) from bone marrow (BM) to the peripheral blood, mainly in the field of 

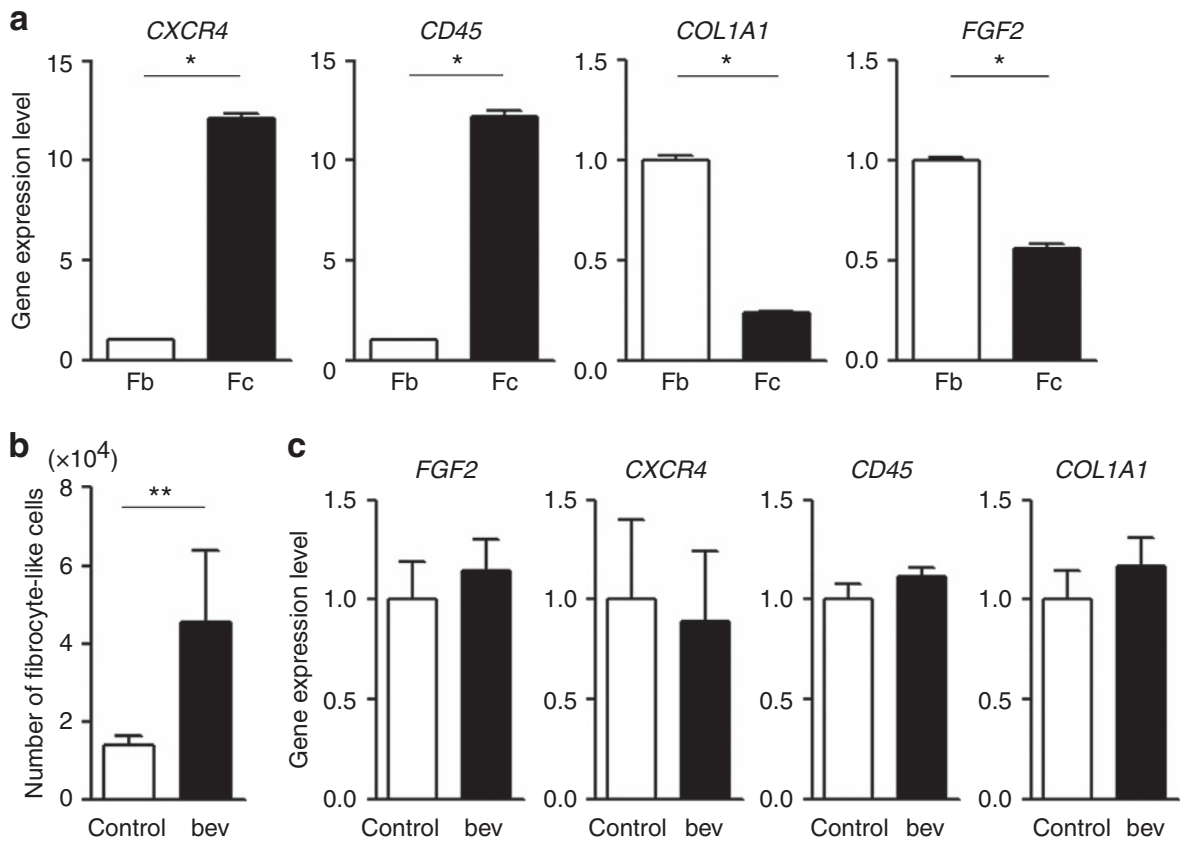

Figure 6 | The isolation and characterization of mouse fibrocyte-like cells from normal mouse lungs or Y-MESO-14 tumours. (a) Fibroblasts (Fb) and fibrocyte-like cells (Fc) were isolated from the normal mouse lungs, and their mRNA expression levels were compared $\left(n=3\right.$ per group). ${ }^{\star} P<0.01$ by Student's t-test. (b,c) Fibrocyte-like cells were isolated from control- or bevacizumab (bev)-resistant tumours. (b) A comparison of the number of isolated fibrocyte-like cells ( $n=6$ per group). (c) A comparison of the mRNA expression in isolated fibrocyte-like cells ( $n=6$ per group). ${ }^{\star \star} P<0.05$ by the MannWhitney-U-test. All data are shown as the means \pm s.e.m.

bone marrow transplantation in non-solid tumours ${ }^{26,27}$. Thus, since we observed that the number of fibrocyte-like cells in the tumour was inhibited by AMD3100 treatment, there is a possibility that its inhibition might be due to the reduction in the production of fibrocyte-like cells from their BM-ancestor cells. To test this hypothesis, we analysed Sca- ${ }^{+}$HSCs in the $\mathrm{BM}$ of bevacizumab- or bevacizumab plus AMD3100-treated mice by flow cytometry. As a result, the addition of AMD3100 in bevacizumab treatment did not affect the number of HSCs in BM (Supplementary Fig. 7) suggesting that the reduced number of fibrocyte-like cells in the tumours treated with AMD3100 was not due to the effects on BM in this model.

Ceradini et al. ${ }^{28}$ have demonstrated that HIF-1-induced CXCL12 expression increases the adhesion, migration and homing of circulating CXCR4 ${ }^{+}$endothelial progenitor cells to ischaemic tissue using a mouse ischaemic model. Because VEGF inhibition produces low oxygen conditions in the tumour, these results supports our data showing that bevacizumab treatment recruits fibrocyte-like cells via the CXCL12-CXCR4 axis, although other mechanisms are supposed to be involved ${ }^{29,30}$. Other than CXCR4-positive population, fibrocytes are reported to have a minor population positive of CCR7 instead of CXCR4 (ref. 30). We performed mouse-specific qPCR analysis using the tumour tissues produced by Y-MESO-14 cells, and found that the expressions of both stromal CXCR4 and CCR7 were increased by bevacizumab treatment, and the expression of CCR7 was significantly lower in the tumour tissue compared with CXCR4 expression, (Supplementary Fig. 8), suggesting that CXCR4-positive population plays a role in bevacizumab resistance. In addition, it is also possible that fibrocyte-like cells proliferate in the tumour tissue instead of recruiting from the blood stream. To confirm whether bone marrow-derived cells (BMDCs) proliferate in the tumour tissue, GFP-positive cells were co-stained with mousespecific Ki67 using the tumour sample from BMT experiment. As a result, only $9.6 \%$ of GFP-positive cells were Ki67-positive, suggesting that the majority of fibrocyte-like cells in the tumour are recruited from the blood (Supplementary Fig. 9).

Pericytes are another important cell type to cover and protect endothelial cells from death induced by VEGF inhibition ${ }^{15,31,32}$. The change in pericyte coverage was also investigated immunohistochemically in our model, but we found that the pericyte coverage was not significantly increased in the bevacizumab-resistant tumours compared with the control tumours (Supplementary Fig. 10).

Fibrocyte-like cell recruitment in human lung cancer tissue. We finally investigated human clinical samples to assess whether there was tumour infiltration of fibrocyte-like cells in human lung cancer. Fibrocyte-like cells $\left(\mathrm{CD} 45^{+} / \mathrm{FSP}^{-1}{ }^{+}\right)$were immunohistochemically detected in surgically resected tumours from lung adenocarcinoma patients. Interestingly, the number of $\mathrm{CD} 45^{+} \%$ FSP- ${ }^{+}$cells was significantly higher in the tumour from patients who received bevacizumab-containing chemotherapy before the surgery compared with the tumour from the patients who received chemotherapy alone or who received no prior therapy (Fig. 8a-c). In addition, the number of FGF2-expressing cells was also significantly increased in the tumours from patients who received bevacizumab-containing chemotherapy (Fig. 8d-f). Most of the FSP-1-positive cells were also FGF2-positive, whereas the CD68positive cells were FGF2-negative (Fig. 8g,h). Furthermore, we coimmunostained FSP-1 and CD3, CD19 or CD68 to verify the possibility that FSP-1-positive cells were lymphocytes or macrophages, and found that these markers did not co-localize with FSP-1 (Supplementary Fig. 11a). CD3-positive cells were detected to a certain extent in human lung cancer tissue although the number of these cells did not change with bevacizumab treatment (Supplementary Fig. 11b). CD19- or CD68-positive cells were minor populations regardless of bevacizumab treatment. Recently, Chung et al. ${ }^{33}$ have demonstrated that interleukin-17 (IL-17) 
a

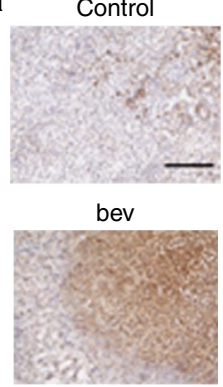

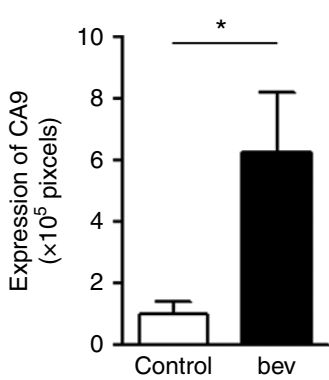

b
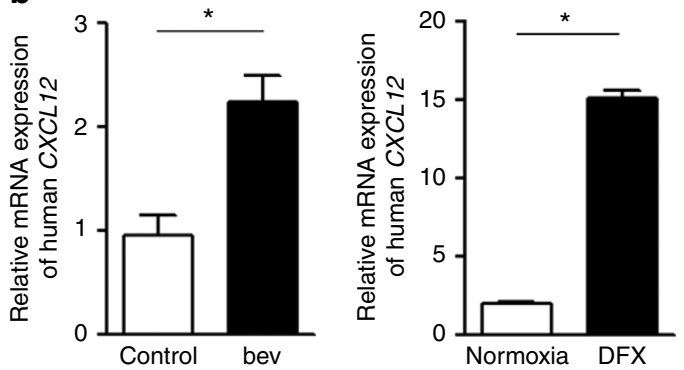

C
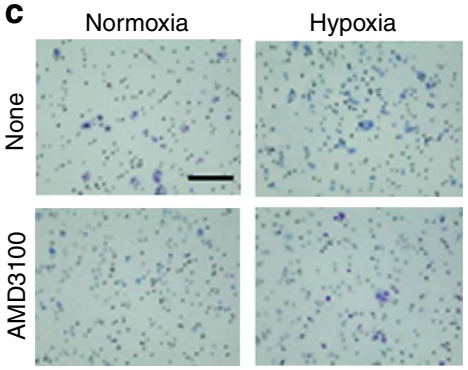

e

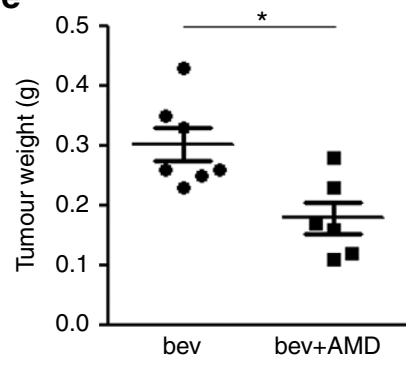

d

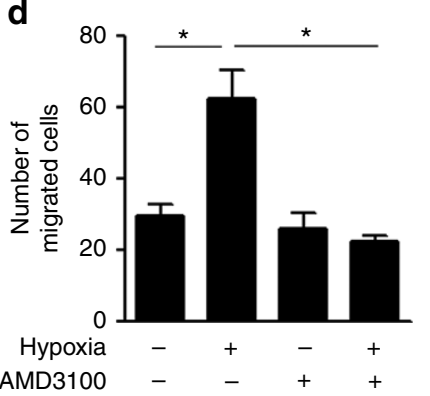

f

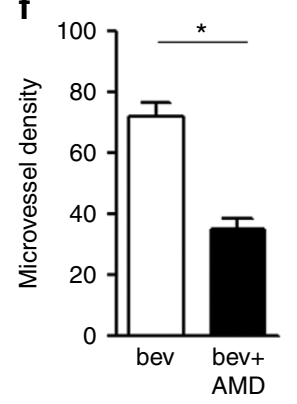

g

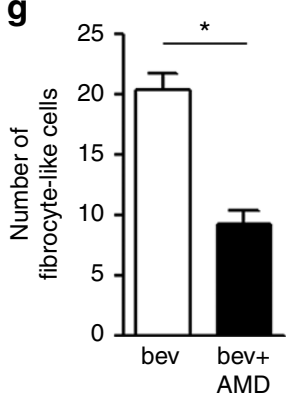

Figure 7 | The involvement of CXCL12-CXCR4 axis in the fibrocyte-like cell-mediated angiogenesis. (a) Assessment of hypoxic lesion in the tumour with immunohistochemistry using anti-CA9 antibody. (left) representative images of the hypoxic lesion stained in brown in the tumour produced by Y-MESO-14 cells treated with or without bevacizumab (bev). Tumours were harvested 28 days after the tumour cell-injection. Scale bar, $200 \mu \mathrm{m}$. (right) Evaluation of CA9-positive area ( $n=6$ per group). ${ }^{\star} P<0.05$ by the Mann-Whitney-U-test. (b) (left) the relative mRNA expression of human (tumour cell) CXCL12 in control- or bevacizumab (bev)-treated Y-MESO-14 tumour tissues ( $n=5$ per group). ${ }^{\star} P<0.01$ by the Mann-Whitney-U-test. Right, the mRNA expression of CXCL12 in Y-MESO-14 cells cultured under normoxia or chemical hypoxia induced by deferoxamine (DFX) in vitro ( $n=3$ per group). ${ }^{\star} P<0.01$ by Student's $t$-test. (c) Representative images of the migrated fibrocyte-like cells in vitro. Fibrocyte-like cells were cultured with or without a CXCR4 inhibitor (AMD3100) in the upper chamber, and those that migrated towards the lower chamber were stained. The lower chamber contained Y-MESO-14 cells cultured under normoxia or chemical hypoxia. Scale bar, $200 \mu \mathrm{m}$. (d) The number of fibrocyte-like cells that migrated under each condition. ${ }^{\star} P<0.05$ by a one-way ANOVA. All in vitro data are representative of duplicate experiments with similar results. (e-g) The effect of CXCR4 inhibition in addition to bevacizumab treatment in vivo. Thoracic tumours (Y-MESO-14) were harvested from mice treated with bevacizumab (bev) or bevacizumab in combination with AMD3100 (AMD). (e) A comparison of the tumour weights. (f) A comparison of MVD. (f) A comparison of the number of fibrocyte-like cells (collagen type $1^{+} / \mathrm{CXCR} 4{ }^{+}$cells) $\left(n=6-7\right.$ per group). ${ }^{\star} P<0.01$ by the Mann-Whitney- $U$-test. All in vivo and in vitro data are shown as the means \pm s.e.m.

promotes tumour resistance to VEGF inhibition by mediating immature myeloid-cell mobilization and recruitment into the tumour microenvironment in mice. The involvement of IL-17 producing cells was also investigated in our clinical samples; however, the number of IL-17-producing cells was not increased in the bevacizumab treatment group in our study (Supplementary Fig. 12). Of note, the number of $\mathrm{CD} 45^{+} / \mathrm{FSP}-1^{+}$cells in the bevacizumab-treated patients was significantly correlated with the number of treatment cycles of bevacizumab (Fig. 8i) and with the vessel length in the tumour (Fig. 8j). Together with the results from mouse models, these findings indicate that fibrocyte-like cells may be key regulatory cells in the tumour microenvironment that are involved in the acquisition of resistance to anti-VEGF therapy through their production of FGF2.

\section{Discussion}

Fibrocytes, which are present in the peripheral circulation as a minor population of leukocytes, were first identified more than a decade ago $^{34}$. Subsequent studies revealed that they are monocyte-derived cells that have features of both macrophages and fibroblasts $22-24,35,36$. Since there is no single specific marker for fibrocytes at present, the combination of intracellular collagen staining and the expression of a hematopoietic marker, such as CD45, plus either CD34 or CXCR4, has been considered to be a sufficiently accurate criterion for identifying fibrocytes $22,23,37$. In some studies, fibrocytes are considered as the cells double positive of CD45 and FSP-1 (refs 38-40). Based on their fibrogenic properties, fibrocytes were previously reported to be involved in the pathogenesis of various fibrotic diseases, such as pulmonary 
a

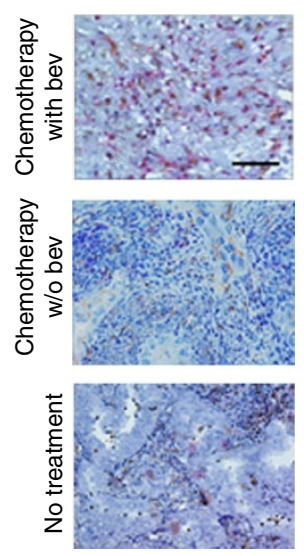

b
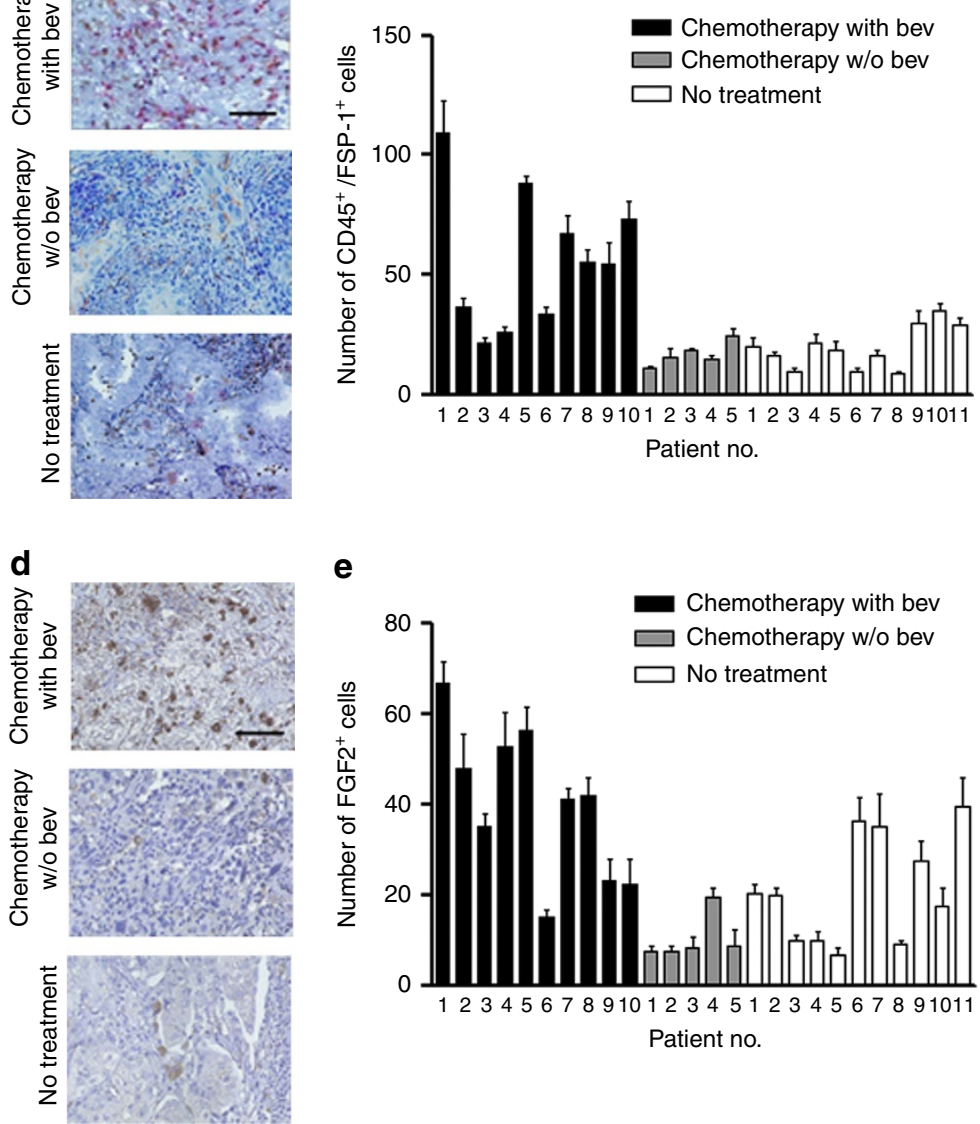

e

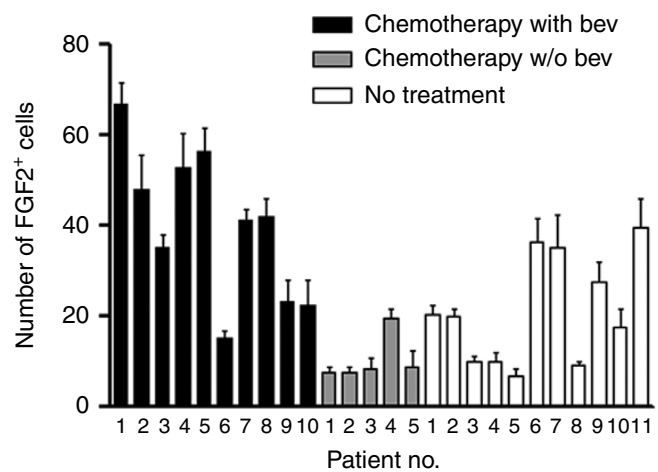

C

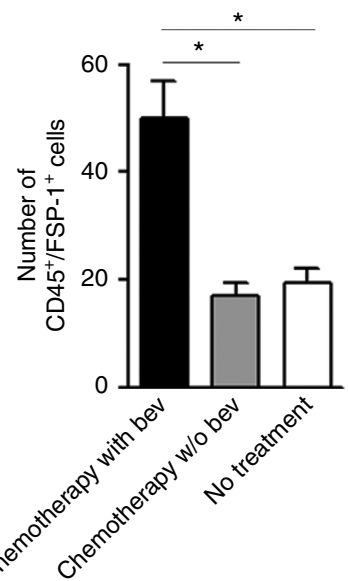

f

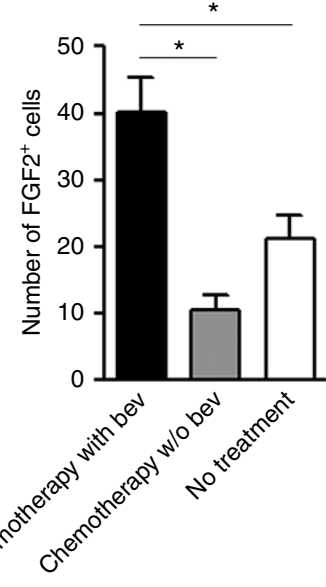

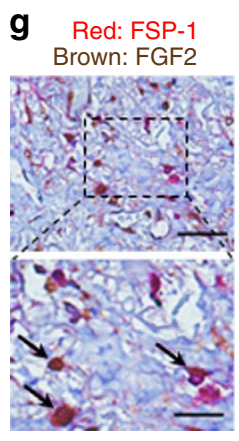
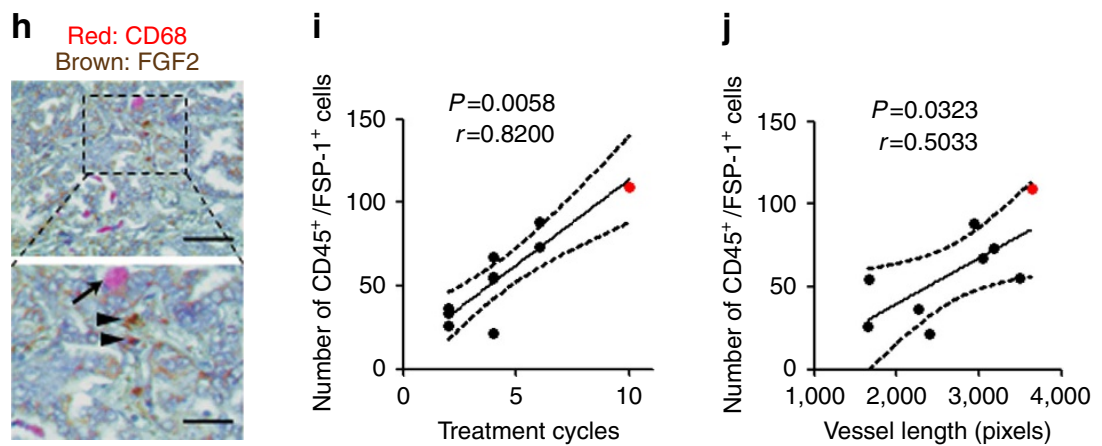

Figure 8 | The recruitment of fibrocyte-like cells in the surgically resected human lung cancer specimens. (a) Representative images of fibrocyte-like cells that were double-positive for CD45 (red) and FSP-1 (brown), in the tumour tissues of patients treated with neoadjuvant chemotherapy containing bevacizumab (bev), chemotherapy without bev or with no prior therapy. Scale bar, $100 \mu \mathrm{m}$. (b) The number of CD45 ${ }^{+} / \mathrm{FSP}-1^{+}$cells in the individual tumour samples. (c) The average number of $\mathrm{CD} 45^{+} / \mathrm{FSP}-1^{+}$cells in each group. ${ }^{\star} P<0.01$ by a one-way ANOVA. (d) Representative images of the FGF2positive cells (brown) in each group. Scale bar, $100 \mu \mathrm{m}$. (e) The number of FGF2-positive cells in the individual tumour samples. (f) The average number of FGF2-positive cells in each group. ${ }^{\star} P<0.01$ by a one-way ANOVA. (g,h) Representative images of the double staining for (g) FSP-1 (red) or (h) CD68 (red) and FGF2 (brown) in the tumour from patient no. 1. (g) The arrows indicate double-positive cells. (h) The arrow indicates a CD68 single-positive cell, and the arrowhead indicates a FGF2 single-positive cell. Scale bars, $100 \mu \mathrm{m}$ (top), $50 \mu \mathrm{m}$ (bottom). (i,j) The correlation of the number of CD45 ${ }^{+} /$FSP- ${ }^{+}$cells in the tumour and (i) the cycles of bevacizumab used and ( $\mathbf{j}$ ) the vessel length in the tumour. Each dot represents an individual tumour sample from patient nos 1-10. The correlation was estimated by Spearman's correlation and a linear regression analysis (the best-fit line is indicated together with the $95 \%$ confidence bands). The red dot indicates patient no. 1, who underwent surgical resection after developing obvious clinical resistance to bevacizumab.

fibrosis, bronchial asthma and cardiovascular disease $e^{41-45}$. In the field of cancer, however, only a small number of studies have reported the existence of fibrocytes in the peripheral blood of patients ${ }^{46}$, and the role of fibrocytes in the pathogenesis of cancer has been unknown, and to the best of our knowledge, their role in drug resistance has not yet been examined.
Although it is complicated to determine the precise identity of monocyte-derived cell populations because of their plasticity and phenotypical overlaps, we applied the term 'fibrocyte-like cells' for collagen type $\mathrm{I}^{+} / \mathrm{CXCR} 4^{+}$(and also $\mathrm{CD}^{+} 5^{+}$in some experiments) cells or $\mathrm{CD} 45^{+} / \mathrm{FSP}-1^{+}$cells, and showed those cell-mediated mechanism of the acquired resistance to 
bevacizumab treatment in both mouse models and human clinical tissue samples. To our knowledge, the previous studies using clinical specimens to investigate the mechanism of the resistance to anti-angiogenic therapy have been focused on the soluble factors present in peripheral blood such as VEGF, placental growth factor, CXCL12 and FGF2 (refs 47-52). Considering that these soluble factors have not been successfully developed as a practical biomarker in clinic, and because the cell-cell interactions play crucial roles in the tumour microenvironment, it seems logical to investigate tissue samples. In our study, not all of the patients who received bevacizumab clinically acquired resistance before the surgery. However, patient no. 1, who had the highest number of fibrocytes and the highest FGF2 expression in the tumour tissue (Fig. 8b,e), underwent surgical resection after developing obvious clinical resistance to bevacizumab. This might support the involvement of fibrocytelike cells in the resistance of human lung cancer to anti-VEGF therapy. In addition, the clinical data also suggest that the mechanism of resistance mediated by fibrocyte-like cells has already been 'switched on' before the development of clinically relevant tumour recurrence. Although it is challenging to obtain surgically removed samples after bevacizumab treatment, given the small number of such samples available, continuous efforts are warranted to clarify the cellular mechanism by which fibrocyte-like cells may mediate the acquired resistance to antiangiogenic therapy.

Various modes of resistance to anti-angiogenic therapy are reviewed in detail by Bergers and Hanahan ${ }^{10}$. According to the current knowledge, the major mechanisms of host-mediated acquired resistance include revascularization subsequent to the upregulation of alternative pro-angiogenic signals; the recruitment of pro-angiogenic cells from the bone marrow and the protection of the vasculature by pericyte coverage. Among the soluble factors that could mediate the angiogenic switch, FGF2 appears to be one of the most intensively reported factor in both the experimental and clinical settings $s^{47,48,53-55}$. With regard to the cells responsible for producing FGF2 in the resistant tumours, the results from our study demonstrated that fibrocyte-like cells may be a major source, in addition to macrophages and the tumour cells themselves.

Tumour angiogenesis, and possibly the re-angiogenesis that develops in the tumours resistant to anti-angiogenesis therapy, can be regulated not only by soluble pro-angiogenic factors but also by the recruitment of various BMDCs that have the capacity to regenerate the blood vessels. Those BMDCs include TAMs, TIE2 ${ }^{+}$ monocytes, VEGFR ${ }^{+}$hemangiocytes and $\mathrm{CD} 11 \mathrm{~b}^{+}$myeloid cells ${ }^{56-61}$. In this study, we demonstrated that the fibrocyte-like cells, but not CD3- or CD19-positive cells, in the tumour microenvironment are involved in the acquisition of resistance to anti-VEGF therapy. As previously reported ${ }^{12,13}$, TAMs do contribute on tumour angiogenesis. There is a possibility that, in addition to fibrocyte-like cells, tumour-infiltrated macrophages may also contribute on angiogenesis in our model, as the number of $\mathrm{CD} 45^{+} / \mathrm{F} 4 / 80^{+}$macrophages was slightly increased with bevacizumab treatment (Fig. 5c). For this, future efforts are warranted to clarify the role of macrophages in the resistance to anti-VEGF therapy in this model. Since we observed in human clinical specimens and in immunodeficient mouse model that F4/ $80^{+}$or $\mathrm{CD}^{+} 8^{+}$cells did not express FGF2 (Figs $3 \mathrm{a}$ and $8 \mathrm{~h}$ ), macrophages may contribute on the acquired resistance via the mechanism(s) other than FGF2-FGFR signalling.

Among the cells that could be able to contribute on the resistance to anti-angiogenesis therapy, $\mathrm{CD} 11 \mathrm{~b}^{+} \mathrm{Gr} 1^{+}$MDSCs were reported to be directly involved in the tumour refractoriness to anti-VEGF therapy in a mouse model ${ }^{14}$. In this study, the number of tumour-infiltrated Gr-1 ${ }^{+}$cells was not changed after
anti-VEGF therapy. However, contribution of MDSCs on acquired resistance to anti-VEGF treatment should not be ignored, as recent reports suggest that some populations of MDSCs (CCR2 ${ }^{+}$or

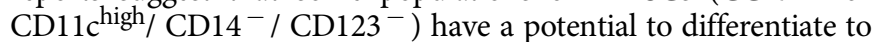
fibrocytes and encourage tumour progression ${ }^{46,62}$. Since these cells have not yet been clearly identified in humans, future technological developments are needed to determine whether these cells exist and are a cause of resistance in humans. There are several potential clinical implications of the present study. First, based on our preclinical data generated using mouse models, dual inhibition of FGF2 and VEGF could provide a more durable response to antiangiogenesis therapy compared with single treatment with bevacizumab. Moreover, it is of interest to ask what other molecules (or cells) are involved after the resistance against FGF2 and VEGF. Second, fibrocyte-like cells could be the key regulator of the resistance to bevacizumab therapy. Thus, it may be instructive to target these cells to more effectively inhibit tumour angiogenesis. Third, fibrocyte-like cells could also be useful as a cellular biomarker for the resistance to bevacizumab therapy. As such, it would be interesting to develop the technology to detect these cells in the peripheral blood to predict the resistance to antiangiogenesis therapy.

In summary, we uncovered that a cell type appears to mediate the resistance to anti-VEGF therapy. It is crucial to focus not only on the soluble factors but also on the responsible cells, to understand the mechanisms of resistance to treatment, and comprehending the mechanism mediated by fibrocyte-like cells might help to develop more effective and durable anti-angiogenesis therapy.

\section{Methods}

Cell lines. The human MPM cell lines, Y-MESO-14 and EHMES-10, were kindly provided by Dr Y. Sekido (Aichi Cancer Center Research Institute, Nagoya, Japan) and Dr H. Hamada (Hiroshima University Graduate School of Health Science, Hiroshima, Japan), respectively. The human NSCLC cell line PC14PE6, and mouse melanoma cell line B16 were kindly provided by Dr I.J. Fidler (M.D. Anderson Cancer Center, TX). The human NSCLC cell line, A549, was purchased from the American Type Culture Collection. Normal HUVECs were purchased from KURABO (Osaka, Japan). These cell lines were authenticated at BEX CO., LTD (Tokyo, Japan) using a Multiplex STR assay. Human thoracic tumour cell lines were maintained in RPMI 1640 medium supplemented with $10 \%$ heat-inactivated fetal bovine serum (FBS), penicillin $\left(100 \mathrm{U} \mathrm{ml}^{-1}\right)$ and streptomycin $\left(50 \mu \mathrm{g} \mathrm{ml}^{-1}\right)$. B16 cells were maintained in DMEM medium supplemented with $10 \%$ heatinactivated FBS, penicillin $\left(100 \mathrm{U} \mathrm{ml}^{-1}\right)$ and streptomycin $\left(50 \mu \mathrm{g} \mathrm{ml}^{-1}\right)$. HUVECs were maintained in EBM-2 (Lonza, Basel, Switzerland). All cells were cultured at $37^{\circ} \mathrm{C}$ in a humidified atmosphere of $5 \% \mathrm{CO}_{2}$ in air.

Reagents. Bevacizumab, an immunoglobulin (Ig) G1 monoclonal antibody targeting VEGF-A, was obtained from Chugai Pharmaceutical (Tokyo, Japan). For in vivo studies, bevacizumab was diluted in phosphate-buffered saline (PBS). BGJ-398, a FGFR-specific inhibitor, was purchased from Chemietek (Indianapolis, IN). For the in vitro studies, $4 \mathrm{mM}$ stock solutions were prepared in DMSO. For the in vivo studies, BGJ-398 was formulated in PEG300/D5W (2:1). FGF2-neutralizing antibody was purchased from R\&D systems (Minneapolis, MN). AMD3100, a selective CXCR4 antagonist, was purchased from Sigma (St Louis, MO). For in vitro studies, $10 \mathrm{mg} \mathrm{ml}^{-1}$ stock solutions were prepared in PBS. SU5416, a VEGFR-specific inhibitor, was purchased from Abcam (Cambridge, MA). For the in vivo studies, SU5416 was formulated in DMSO. An anti-mouse IL-2 receptor $\beta$-chain monoclonal antibody, TM- $\beta 1$, was supplied by Drs M. Miyasaka and T. Tanaka (Osaka University, Osaka, Japan).

Animals. Six-weeks-old male athymic BALB/c nude mice, severe combined immunodeficient (SCID) mice and C57BL/6 mice were obtained from Charles River Japan Inc. (Shiga, Japan) or CLEA Japan (Tokyo, Japan), respectively. Sixweeks-old male C57BL/6-BALB/c-nu/nu-EGFP mice were obtained from SLC Japan Inc. (Shizuoka, Japan). Mice were maintained under specific pathogen-free conditions throughout this study. All experiments were performed in accordance with the guidelines established by the Tokushima University Committee on Animal Care and Use. At the end of each in vivo experiment, the mice were anaesthetized with isoflurane and euthanized humanely by cutting the subclavian artery. All experimental protocols were reviewed and approved by the animal research committee of The University of Tokushima, Japan. 
In vivo orthotopic implantation models of human MPM cells. For orthotopic implantation, SCID mice were anaesthetised with tribromoethanol and had their right chest wall shaved. After sterilization of the chest wall with $70 \%$ ethanol, their right chest skin was cut, and their parietal pleura were exposed. They were then injected with Y-MESO-14 or EHMES- 10 cells $\left(1.0 \times 10^{6}\right.$ cells per mouse) suspended in $0.1 \mathrm{ml}$ PBS into the thoracic cavity through the parietal pleura using a $27 \mathrm{G}$ needle ${ }^{63}$. To determine the therapeutic efficacy of bevacizumab against intrathoracic implanted tumour tissue, the mice were treated twice a week with $10 \mu \mathrm{g}$ per mouse bevacizumab by intraperitoneal injection, beginning 1 week after tumour cell inoculation. The mice were killed humanely under anaesthesia, and the thoracic tumours were removed and weighed 21, 28, 35, 42 days (Y-MESO-14) or 28 and 65 days (EHMES-10) after tumour cell inoculation. Whole tumour tissues were weighted, and the weights were compared between bevacizumab-treated group and vehicle-treated group. The pleural effusion was harvested using a $1 \mathrm{ml}$ syringe. To determine the effects of bevacizumab on survival, mice were treated twice a week with $10 \mu \mathrm{g}$ per mouse bevacizumab or vehicle control from day 7 until they became moribund. To examine the effects of combination treatment with bevacizumab and BGJ-398, the mice were treated once daily with $200 \mu \mathrm{g}$ per mouse BGJ-398 or vehicle control by oral gavage from days 21 to 35 . To determine the effects of combination treatment with bevacizumab and FGF2-neutralizing antibody, the mice were treated with $20 \mu \mathrm{g}$ per mouse goat anti-FGF2 antibody or control goat IgG (R\&D systems, Minneapolis, MN) by subcutaneous injection on days 7 and 14 in combination with continuous bevacizumab treatment stated above. To examine the effects of combination treatment with bevacizumab and a CXCR4 antagonist (AMD3100), the mice were treated once daily with $5 \mathrm{mg} \mathrm{kg}^{-1}$ per mouse AMD3100 or vehicle control by subcutaneous injection from days 7 to 42 . Considering the inhibitory effect of AMD3100 in the anti-tumour immune response via NK cells, all mice were pretreated with TM- $\beta 1$ ( $1 \mathrm{mg}$ per $0.3 \mathrm{ml}$ PBS per mouse) by intraperitoneal injection to deplete NK cells two days before tumour inoculation ${ }^{64}$. The mice were killed humanely under anaesthesia, and thoracic tumours were removed on day 42 . Whole tumour tissues were weighted, and the weights were compared between the groups treated with combination therapy and bevacizumab monotherapy.

In vivo lung metastasis model. To establish the lung metastasis model, nude mice were intravenously inoculated via the tail vein with PC14PE6 or A549 tumour cells $\left(1.0 \times 10^{6}\right.$ cells per mouse) suspended in $0.2 \mathrm{ml}$ of PBS ${ }^{65}$. Mice were treated with bevacizumab with the same procedure used in the orthotopic models.

GFP bone marrow chimeric mouse model. Bone marrow cells from C57BL/6$\mathrm{BALB} / \mathrm{c}-\mathrm{nu} / \mathrm{nu}$-EGFP mice were extracted from the tibias and femurs and transplanted by tail-vein injection $\left(5.0 \times 10^{6}\right.$ cells per mouse) into irradiated (two doses of $3.5 \mathrm{~Gy}, 3 \mathrm{~h}$ apart) nude mice. Three weeks after the BMT, the mononuclear cells in the peripheral blood of recipient mice were screened by Keyence BZ-9000 microscopy (Keyence, Tokyo, Japan), and were confirmed to be over $90 \%$ GFPpositive. The mice were pretreated with TM- $\beta 1$ ( $1 \mathrm{mg}$ per $0.3 \mathrm{ml}$ PBS per mouse) by intraperitoneal injection to deplete NK cells three weeks after the BM transplant. Two days later, the mice were orthotopically inoculated with Y-MESO-14 tumour cells $\left(1.0 \times 10^{6}\right.$ cells per mouse $)$ and treated twice a week with $10 \mu \mathrm{g}$ per mouse bevacizumab or vehicle control by intraperitoneal injection from day 7 . The mice were killed humanely under anaesthesia and thoracic tumours were removed 28 days after tumour cell inoculation.

In vivo subcutaneous implantation model of B16 cells. Mouse melanoma cell line, B16 cells $\left(1.0 \times 10^{6}\right.$ cells per mouse) suspended in $0.1 \mathrm{ml}$ PBS were subcutaneously inoculated to the right flank of C57BL/6 mice. Tumour size was measured by vernier caliper at least three times a week. Volume $=a b^{2} 2^{-1}$ ( $a$, long diameter; $b$, short diameter). To determine the effect of SU5416 on tumour growth, the mice were treated once a day with SU5416 (5 or $10 \mathrm{mg}$ per $\mathrm{kg}$ per mouse) or DMSO by intraperitoneal injection from day 5 until they became moribund. When the average of tumour size reached $5000-6000 \mathrm{~mm}^{3}$, the mice were killed humanely and the tumours were resected for the further analyses. To examine the effects of combination treatment with SU5416 and BGJ-398, the mice were treated once daily with $200 \mu \mathrm{g}$ per mouse BGJ-398 or vehicle control by oral gavage from day 5 , in addition to SU5416 treatment.

Immunofluorescence of mouse tumour tissues. The excised tumour tissues from the model mice were placed into OCT compound (Sakura Finetechnical Co., Tokyo, Japan) and snap-frozen. Frozen tissue sections ( $8 \mu \mathrm{m}$ thick) were fixed with $4 \%$ paraformaldehyde solution in PBS and used for the identification of fibrocytelike cells using a goat anti-COL1A1 polyclonal antibody (1:100 dilution, Santa Cruz, Dallas, TX), rabbit anti-FSP-1 polyclonal antibody (1:100 dilution, Neomarkers/Lab Vision Corporation, Fremont, CA), rat anti-CXCR4 monoclonal antibody (1:100 dilution, 247506, R\&D systems, Minneapolis, MN) and rat antiCD45 monoclonal antibody (1:50 dilution, 30-F11, BD Pharmingen, Franklin Lakes, NJ). To detect macrophages, a rat anti-CD68 monoclonal antibody (1:100 dilution, ED1, Serotec, Oxford, UK) and rat anti-F4/80 monoclonal antibody (1:100 dilution, A3-1, Serotec, Oxford, UK) were used. To identify MDSCs, a rat anti-Gr-1 monoclonal antibody (1:50 dilution, RB6-8C5, BD Pharmingen, Franklin Lakes, NJ) was used. To detect T cells and B cells, a rat anti-CD3 monoclonal antibody (1:150 dilution, 17A2, BD Pharmingen, Franklin Lakes, NJ) and a rat anti-CD19 monoclonal antibody (1:150 dilution, 1D3, BD Pharmingen, Franklin Lakes, NJ) were used, respectively. To detect fibroblasts, a goat anti- $\alpha$ SMA polyclonal antibody (1:150 dilution, Abcam, Cambridge, MA) was used. To determine the expression of FGF2 in the above-mentioned cells, a rabbit anti-FGF2 polyclonal antibody (1:100 dilution, Santa Cruz, Dallas, TX) or goat anti-FGF2 polyclonal antibody (1:50 dilution, Sigma, St Louis, MO) were used. A rat antiCD31/PECAM-1 monoclonal antibody (1:150 dilution, MEC 13.3, BD Pharmingen, Franklin Lakes, NJ) and rabbit anti-NG2 polyclonal antibody (1:150 dilution Millipore, Billerica, MA) were used, respectively, to detect endothelial cells and pericytes. To detect GFP-positive cells, we used a rabbit anti-GFP monoclonal antibody (1:75 dilution, D5.1, Cell Signaling Technology, Danvers, MA) or goat anti-GFP polyclonal antibody (1:50 dilution, Santa Cruz, Dallas, TX). To detect cell proliferation, rabbit anti-mouse Ki67 antibody (1:150 dilution, D3B5, Cell Signaling Technology, Danvers, MA) was used. Alexa488, 594 and 647-labelled secondary antibodies (1:250 dilution, Invitrogen, Carlsbad, CA) were used for immunofluorescent detection. Nuclei were counterstained with DAPI (blue). In each slide, the number of positive cells was counted under a fluorescent microscope at a $\times 200$ magnification. The pericyte coverage of the tumour vasculature was determined by performing double staining for CD31 and NG-2. The expression levels of FGF2 in the cells in the tumour microenvironment were determined by performing double staining for FGF2 and markers of tumour microenvironment cells, such as collagen type I, CXCR4, CD45, CD68, F4/80, Gr-1, CD3, CD19 or aSMA, and double positive cells were evaluated at a $\times 400$ magnification. The expression of FGF2 in fibrocyte-like cells was determined by performing triple staining for FGF2, collagen type I and CXCR4 at a $\times 800$ magnification. In the GFP bone marrow chimeric mouse model, the percentages of the GFP-positive areas were analysed using the Image J software program (National Institutes of Health, Bethesda, MD) at a $\times 200$ magnification. To determine the bone marrowderived fibrocyte-like cells, triple staining of collagen type I, CXCR4 and GFP was performed at a $\times 800$. The expression levels of FGF2 or cell proliferation in BMDCs were determined by performing double staining for FGF2 or Ki67 and GFP at a $\times 400$. These images were acquired by an Olympus BX61 fluorescence light microscope (Olympus, Tokyo, Japan). The antibodies used in this study are listed in Supplementary Table 1.

Immunohistochemical studies of mouse tumour tissue. Frozen tissue sections ( $8 \mu \mathrm{m}$ thick) of tumours were fixed with $4 \%$ paraformaldehyde solution in PBS. To detect endothelial cells, rat anti-CD31/PECAM-1 monoclonal antibody (1:100 dilution, MEC 13.3, BD Pharmingen, Franklin Lakes, NJ) was used. To determine the hypoxia of tumour tissue, rabbit anti-CA9 polyclonal antibody (1:1,000 dilution, Novus Biologicals, Littleton, CO) was used. Appropriate secondary antibodies conjugated with peroxidase (ready to use, Nichirei, Tokyo, Japan) and the 3,3'-diaminobenzidine tetrahydrochloride (DAB) Liquid System (DAKO, Carpinteria, CA) were used to detect the immunostaining. Nuclei were counterstained with hematoxylin (DAKO). MVD (the number of $\mathrm{CD} 31^{+}$structures per HPF) was evaluated at a $\times 200$ magnification ${ }^{15,53,66}$. The CA9-positive areas were calculated using the Image J software program (National Institutes of Health, Bethesda, MD) at a $\times 200$ magnification. Images were acquired by Keyence BZ-9000 microscopy (Keyence, Tokyo, Japan).

Flow cytometry. The tumour tissues collected from Y-MESO-14 tumour-bearing SCID mice were suspended to provide single cells on day 28 after tumour cellinoculation. To identify fibrocyte-like cells, PE-Cy7 conjugated antibodies to CD45 (1:50, 30-F11, eBioscience, San Diego, CA), Fluorescein conjugated anti-CXCR4 (1:10 dilution, 247506, R\&D systems, Minneapolis, MN), and Biotin conjugated anti-COL1A1 (1:100 dilution, Rockland, Limerick, PA) were used. To detect Biotin conjugated antibody, PE-Streptavidin (1:1000 dilution, BD Pharmingen, Franklin Lakes, NJ) was used. FITC conjugated anti-CD45 (1:50 dilution, 30-F11, BD Pharmingen, Franklin Lakes, NI) and PE conjugated antibodies to F4/80 (1:10 dilution, BM8, Biolegend, San Diego, CA) or Gr-1 (1:10 dilution, RB6-8C5, BD Pharmingen, Franklin Lakes, NJ) were used to detect macrophages or MDSCs, respectively. To determine the effect of AMD3100 on HSCs in bone marrow, the bone marrow cells were harvested from the tibias and femurs of tumour (Y-MESO14)-bearing SCID mice on day 42 after the inoculation of tumour cells. The mice were treated once daily with AMD3100 $\left(5 \mathrm{mg} \mathrm{kg}^{-1}\right.$ per mouse) or vehicle control by subcutaneous injection from days $7-42$, in addition to bevacizumab treatment. To identify HSCs, PE conjugated antibody to Sca-1 (1:10 dilution, D7, BD Pharmingen, Franklin Lakes, NJ) was used. The stained cells were analysed by flow cytometry using a BD LSRFortessa (BD Bioscience, San Diego, CA) for acquisition and FlowJo software (Treestar Inc, Ashland, OR) for analysis.

Patient samples. Paraffin-embedded sections from 26 lung adenocarcinoma patients (surgery alone: $n=11$, chemotherapy without bevacizumab: $n=5$, chemotherapy with bevacizumab: $n=10$ ) were provided by The University of Tokushima (Tokushima, Japan), Fukui-ken Saiseikai Hospital (Fukui, Japan), Municipal Tsuruga Hospital (Fukui, Japan) and Kagawa University (Kagawa, Japan). Informed consent was obtained from all patients, and the protocol was approved by the Institutional Review Board (IRB) of Tokushima University Hospital (No. 1750) and then by the IRB of each of the participating institutions. 
Immunohistochemical studies of the patient samples. To detect fibrocyte-like cells in the clinical specimens, the paraffin-embedded tissues $(4 \mu \mathrm{m}$ thick) were stained with a rabbit anti-FSP-1 polyclonal antibody (1:100 dilution, Neomarkers/ Lab Vision Corporation, Fremont, CA) and mouse anti-CD45 monoclonal antibody (1:50 dilution, 136-4B5, Cell Signaling Technology, Danvers, MA). To detect the FGF2-positive cells, a goat anti-FGF2 polyclonal antibody (1:50 dilution, Sigma, St Louis, MO) was used. We used a goat anti-IL-17 polyclonal antibody to detect Th17 cells (1:50 dilution, R\&D systems, Minneapolis, MN). A mouse anti-CD68 monoclonal antibody (ready to use, PG-M1, Nichirei, Tokyo, Japan) was used to detect macrophages. A mouse anti-CD3 monoclonal antibody (1:50 dilution, F7.2.38, Dako, Carpinteria, CA) was used to detect T-cells. To detect the B cells, mouse antiCD19 monoclonal antibody (1:50 dilution, LE-CD19, Dako, Carpinteria, CA) was used. These sections were re-incubated with appropriate secondary antibodies conjugated with peroxidase or alkaline phosphatase (ready to use, Nichirei, Tokyo, Japan). Immunoreactivity was detected by using the DAB Liquid System or new fuchsin (Nichirei, Tokyo, Japan), and samples were counterstained with hematoxylin. The number of positive cells was counted at a $\times 400$ magnification. The expression levels of FGF2 in fibrocyte-like cells or macrophages were determined by performing double staining for FGF2 and FSP-1 or CD68, respectively, at a $\times 400$ or $\times 800$ magnification. Images were acquired by Keyence BZ-9000 microscopy (Keyence, Tokyo, Japan). To detect endothelial cells, CD31 immunohistochemistry was performed using Leica Bond-Max (Leica, Bannockburn, IL) and Bond Polymer Refine Detection (Leica, Bannockburn, IL) according to the manufacturer's protocols. A mouse anti-human CD31 monoclonal antibody (1:50 dilution, JC70A, Dako, Carpinteria, CA) was used as the primary antibody. To determine the microvessel length in tumour tissue, the major axis of vessels was measured by using the BZ-9000 analytical software program (Keyence, Tokyo, Japan).

qRT-PCR. Total RNA was extracted from the tumours using an RNeasy Mini Kit (Qiagen, Valencia, CA), and was reverse-transcribed to cDNA using a High Capacity cDNA Reverse Transcription Kit (Applied Biosystems, Carlsbad, CA) according to the manufacturer's instructions. RT-PCR was performed using the CFX96 real-time PCR system (Bio-Rad, Hercules, CA) or the SYBR Premix Ex Taq (TAKARA, Kyoto, Japan). Human RPL27 and mouse RPS29 mRNA were used as housekeeping genes, and quantification was performed using the $\Delta \Delta \mathrm{Ct}$ method. The specific PCR primer pairs used for each studied gene are shown in Supplementary Table 2 .

Detection of protein expression in mouse tumour tissue. Y-MESO-14 tumour tissues were homogenized in T-PER (Pierce, Rockford, IL) containing phosphatase and protease inhibitor cocktails (Roche, Basel, Switzerland). The concentrations of proteins were determined using a Bio-Rad Protein Assay Kit (Bio-Rad, Richmond, CA). Samples of $500 \mathrm{\mu g} \mathrm{ml}^{-1}$ of total proteins and pleural effusion from tumourbearing mice were used to detect the concentrations of human VEGF, mouse VEGF, FGF1 and FGF2 by using ELISA kits (R\&D Systems, Minneapolis, MN) according to the manufacturer's instructions.

Induction of chemical hypoxia in vitro. Y-MESO-14 tumour cells were plated in 24-well plates with $10 \%$ FBS containing DMEM and were incubated for $24 \mathrm{~h}$. After the incubation, $100 \mu \mathrm{M}$ of a hypoxia-mimetic, DFX mesylate (DFX, Sigma, St Louis, MO), was added to each well, and the cells were incubated for another $24 \mathrm{~h}$ to mimic the hypoxic condition. Total RNA was extracted from the hypoxic tumour cells and the gene expression levels were compared with those in tumour cells incubated under normoxic conditions.

Isolation of human fibrocyte-like cells. Human fibrocyte-like cells were isolated according to previously published methods ${ }^{67}$. Mononuclear cells were isolated from the peripheral blood of healthy volunteers using Ficoll density centrifugation $^{68}$. The isolated cells were cultured in DMEM supplemented with $20 \%$ FBS, penicillin and streptomycin on bovine fibronectin (R\&D systems, Minneapolis, MN)-coated $150 \mathrm{~mm}$ cell culture dishes (BD Pharmingen, Franklin Lakes, NJ). The medium was changed twice a week. After 7-10 days, the media was aspirated and washed with sterile PBS three times. The adherent cells were determined to be circulating fibrocyte-like cells by a flow cytometric analysis and immunostaining ${ }^{67}$. Informed consent was obtained from all volunteers, and the protocol was approved by the IRB of Tokushima University Hospital (No. 1586).

Purification of fibrocyte-like cells from mouse lung. Murine fibrocyte-like cells were isolated according to previously published methods ${ }^{69}$. Murine lungs removed from male SCID mice were minced with scissors and incubated with DMEM including $1 \mathrm{mg} \mathrm{ml}^{-1} \mathrm{BSA}$ (Sigma, St Louis, MO), $1 \mathrm{mg} \mathrm{ml}^{-1}$ collagenase IV (Roche, Basel, Switzerland) and $100 \mu \mathrm{g} \mathrm{ml}^{-1}$ DNase 1 (Sigma, St Louis, MO) for $1 \mathrm{~h}$ at $37^{\circ} \mathrm{C}$. The single cell suspensions from whole lungs were incubated in DMEM supplemented with 20\% FBS in $100 \mathrm{~mm}$ fibronectin-coated dishes. When cells reached $70 \%$ confluence, they were passaged using trypsin digestion. After 7 days, the trypsinized cells were incubated with anti-CD45 Abs coupled to magnetic beads (Miltenyi Biotech, Auburn, CA) for $15 \mathrm{~min}$ on ice. The labelled cells were separated into $\mathrm{CD}_{4} 5^{+}$(fibrocyte-like cells) and $\mathrm{CD} 45^{-}$(fibroblasts) populations using an Auto MACS instrument (Miltenyi Biotech, Auburn, CA) according to the manufacturer's instructions.

Purification of tumour-infiltrated fibrocyte-like cells. The tumour tissues collected from Y-MESO-14 orthotopic tumour-bearing SCID mice were minced and suspended to provide single cells on day 28 after inoculation in the same way described above. The number of whole cells was counted using a hemocytometer, and $3 \times 10^{6}$ suspended cells were labelled with anti-mouse CD45 magnetic beads, and the $\mathrm{CD} 45^{+}$cells were isolated using an Auto MACS instrument. Mouse $\mathrm{CD}_{4}{ }^{+}$cells were plated in fibronectin-coated $100 \mathrm{~mm}$-diameter dishes and incubated for seven days. The adherent cells were considered to be tumour-associated fibrocyte-like cells. The number of the cells was counted using a hemocytometer, and the total RNA was extracted.

Cell migration assay for fibrocyte-like cells. Y-MESO-14 tumour cells were plated in 24 -well plates $\left(5 \times 10^{4}\right.$ cells per $500 \mu \mathrm{l}$ per well) with $10 \%$ FBS containing DMEM and were incubated for $24 \mathrm{~h}$. After the incubation, the medium was changed to $0.1 \%$ FBS containing DMEM with or without $100 \mu \mathrm{M}$ DFX, and cells were incubated for another $24 \mathrm{~h}$. The migration assay was performed using $8 \mu \mathrm{m}$ pore size cell culture inserts (BD Biosciences, San Jose, CA). Purified human fibrocyte-like cells were plated in fibronectin-coated $100 \mathrm{~mm}$-diameter dishes, and were incubated with DMEM supplemented with $10 \%$ FBS for $24 \mathrm{~h}$. Then, the medium was changed to $0.1 \%$ FBS containing DMEM in the presence or absence of $5 \mu \mathrm{g} \mathrm{ml}^{-1}$ of the CXCR4 antagonist, AMD3100, and the cells were incubated for $1 \mathrm{~h}$. After this incubation, the fibrocyte-like cells $\left(3 \times 10^{5}\right.$ cells per $100 \mu \mathrm{l}$ per well $)$ were added to the inside of the cell culture inserts, and the inserts were put on the Y-MESO-14 tumour cells cultured under the conditions described above. After a $3 \mathrm{~h}$ incubation, the cells that had migrated to the bottom surface of the filter were stained using Diff-Quik reagents I and II (Baxter, Morton Grove, IL) and were counted in six randomly selected fields on each filter under a microscope at a $\times 200$ magnification.

Cell proliferation assay of HUVECs. Secondary cultures of human fibrocyte-like cells were plated into 24 -well fibronectin-coated plates $\left(1 \times 10^{5}\right.$ cells per well $)$ with the culture medium described above. After $24 \mathrm{~h}$, the medium was changed to $400 \mu \mathrm{l}$ of $0.1 \%$ FBS containing DMEM, and the cells were incubated for an additional $48 \mathrm{~h}$. The conditioned medium was collected and stored at $-80^{\circ} \mathrm{C}$ for further experiments. The proliferation of HUVECs was measured using the MTT (3-[4, 5-dimethylthiazol-2-yl]-2, 5-diphenyl tetrazolium) dye reduction method. HUVECs $\left(2 \times 10^{3}\right.$ cells per $\left.100 \mu \mathrm{l}\right)$ were plated into each well of 96-well type 1 collagen coated-plates (Iwaki, Tokyo, Japan) in 0.1\% FBS containing DMEM and were incubated for $24 \mathrm{~h}$, then $100 \mu \mathrm{l}$ of the culture supernatant from fibrocyte-like cells was added. Recombinant human VEGF protein $\left(10 \mathrm{ng} \mathrm{ml}^{-1}\right.$, R\&D systems, Minneapolis, MN) or FGF protein (10 $\mathrm{ng} \mathrm{ml}^{-1}$, PeproTech, Rocky Hill, NJ) containing medium was added as a positive control. Then, bevacizumab ( 1 or $\left.0.1 \mu \mathrm{g} \mathrm{ml}^{-1}\right)$ or BGJ-398 (100 or $\left.10 \mathrm{nM}\right)$ was added, and the cells were incubated for an additional $72 \mathrm{~h}$. After the incubation, $50 \mu \mathrm{l}$ of stock MTT solution ( $2 \mathrm{mg} \mathrm{ml}^{-1}$, Sigma, St Louis, MO) was added to all of the wells, and the cells were incubated for $2 \mathrm{~h}$ at $37^{\circ} \mathrm{C}$. The media containing the MTT solution was removed, and the dark blue crystals were dissolved by adding $100 \mu$ l of DMSO. The absorbance was measured with a SUNRISE Remote R microplate reader (Tecan, Mannedorf, Switzerland) at test and reference wavelengths of 550 and $630 \mathrm{~nm}$, respectively.

Microarray analysis. Total RNA was extracted from snap-frozen Y-MESO-14 tumour tissues implanted in the SCID mice with or without bevacizumab treatment by using a RNeasy Mini Kit (Qiagen, Valencia, CA). The quality of the RNA samples were confirmed using an Agilent 2100 Bioanalyzer (G2938C, Agilent Technologies, Palo Alto, CA) employing an Agilent RNA 6000 Nano LabChip Kit (5067-1511, Agilent Technologies, Palo Alto, CA). A total of $200 \mathrm{ng}$ of total RNA was amplified and labelled using the Agilent Low Input Quick Amp Labelling Kit, One-Color kit (5190-2305, Agilent Technologies, Palo Alto, CA), and labelled RNA was hybridized to Agilent Whole Human Genome Gene Expression $4 \times 44 \mathrm{~K}$ ver 2.0 Micro Arrays (Agilent Technologies, Palo Alto, CA) for $17 \mathrm{~h}$ at $65^{\circ} \mathrm{C}$. The Agilent Feature Extraction Image Analysis Software program (Version 10.7.3, Agilent Technologies, Palo Alto, CA) was used to extract data from the raw microarray image files. The data visualization and analysis were performed using the Gene Spring GX (Version 12.5, Agilent Technologies, Palo Alto, CA) software program. Genes with an altered expression with more than 2-fold were identified on each sample. The microarray data were deposited in the NCBI Gene Expression Omnibus (GEO) under accession code GSE59476.

Statistical analyses. The data are presented as the means \pm s.e.m. The statistical analyses were performed using Student's $t$-test for unpaired samples, the MannWhitney- $U$-test or a one-way ANOVA, followed by Tukey's multiple-comparison post-hoc test, were also used as appropriate. Values of $P<0.05$ were considered to be statistically significant. 


\section{References}

1. Folkman, J. Angiogenesis: an organizing principle for drug discovery? Nat. Rev. Drug Discov. 6, 273-286 (2007).

2. Carmeliet, P. \& Jain, R. K. Molecular mechanisms and clinical applications of angiogenesis. Nature 473, 298-307 (2011).

3. Ferrara, N., Gerber, H. P. \& LeCouter, J. The biology of VEGF and its receptors. Nat. Med 9, 669-676 (2003).

4. Ferrara, N. \& Davis-Smyth, T. The biology of vascular endothelial growth factor. Endocr. Rev. 18, 4-25 (1997).

5. Sandler, A. et al. Paclitaxel-carboplatin alone or with bevacizumab for nonsmall-cell lung cancer. N. Engl. J. Med. 355, 2542-2550 (2006)

6. Hurwitz, H. et al. Bevacizumab plus irinotecan, fluorouracil, and leucovorin for metastatic colorectal cancer. N. Engl. J. Med. 350, 2335-2342 (2004).

7. Montero, A. J. \& Vogel, C. Fighting fire with fire: rekindling the bevacizumab debate. N. Engl. J. Med. 366, 374-375 (2012).

8. Rapisarada, A. \& Melillo, G. Overcoming disappointing results with antiangiogenic therapy by targeting hypoxia. Nat. Rev. Clin. Oncol. 9, 378-390 (2012).

9. Ebos, J. M. \& Kerbel, R. S. Antiangiogenic therapy: impact on invasion, disease progression, and metastasis. Nat. Rev. Clin. Oncol. 8, 210-221 (2011).

10. Bergers, G. \& Hanahan, D. Modes of resistance to anti-angiogenic therapy. Nat. Rev. Cancer 8, 592-603 (2008).

11. De Bock, K., Mazzone, M. \& Carmeliet, P. Antiangiogenic therapy, hypoxia, and metastasis: risky liaisons, or not? Nat. Rev. Clin. Oncol. 8, 393-404 (2011).

12. Murdoch, C., Muthana, M., Coffelt, S. B. \& Lewis, C. E. The role of myeloid cells in the promotion of tumour angiogenesis. Nat. Rev. Cancer 8, 618-631 (2008).

13. Lin, E. Y. et al. Macrophages regulate the angiogenic switch in a mouse model of breast cancer. Cancer Res. 66, 11238-11246 (2006).

14. Shojaei, F. et al. Tumor refractoriness to anti-VEGF treatment is mediated by CD11b + Gr1 + myeloid cells. Nat. Biotechnol. 25, 911-920 (2007).

15. Cascone, T. et al. Upregulated stromal EGFR and vascular remodeling in mouse xenograft models of angiogenesis inhibitor-resistant human lung adenocarcinoma. J. Clin. Invest. 121, 1313-1328 (2011).

16. Bottsford-Miller, J. N., Coleman, R. L. \& Sood, A. K. Resistance and escape from antiangiogenesis therapy: clinical implications and future strategies. J. Clin. Oncol. 30, 4026-4034 (2012).

17. Li, Q. et al. The therapeutic efficacy of anti-vascular endothelial growth factor antibody, bevacizumab, and pemetrexed against orthotopically implanted human pleural mesothelioma cells in severe combined immunodeficient mice. Clin. Cancer Res. 13, 5918-5925 (2007).

18. Ikuta, K. et al. E7080, a multi-tyrosine kinase inhibitor, suppresses the progression of malignant pleural mesothelioma with different proangiogenic cytokine production profiles. Clin. Cancer Res. 15, 7229-7237 (2009).

19. Yano, S. et al. Production of experimental malignant pleural effusions is dependent on invasion of the pleura and expression of vascular endothelial growth factor/vascular permeability factor by human lung cancer cells. Am. J. Pathol. 157, 1893-1903 (2000).

20. Yano, S. et al. Multifunctional interleukin-1beta promotes metastasis of human lung cancer cells in SCID mice via enhanced expression of adhesion-, invasionand angiogenesis-related molecules. Cancer Sci. 94, 244-252 (2003).

21. Guagnano, V. et al. Discovery of 3-(2,6-dichloro-3,5-dimethoxy-phenyl)-1-\{6-[4(4- ethyl-piperazin-1-yl)-phenylamino]-pyrimidin-4-yl\}-1-methyl-urea (NVPBGJ398), a potent and selective inhibitor of the fibroblast growth factor receptor family of receptor tyrosine kinase. J. Med. Chem. 54, 7066-7083 (2011).

22. Reilkoff, R. A., Bucala, R. \& Herzog, E. L. Fibrocytes: emerging effector cells in chronic inflammation. Nat. Rev. Immunol. 11, 427-435 (2011).

23. Pilling, D., Fan, T., Huang, D., Kaul, B. \& Gomer, R. H. Identification of markers that distinguish monocyte-derived fibrocytes from monocytes, macrophages, and fibroblasts. PLoS ONE 4, e7475 (2009).

24. Hartlapp, I. et al. Fibrocytes induce an angiogenic phenotype in cultured endothelial cells and promote angiogenesis in vivo. FASEB J. 15, 2215-2224 (2001).

25. De Clercq, E. The bicyclam AMD3100 story. Nat. Rev. Drug Discov. 2, 581-587 (2003).

26. Broxmeyer, H. E. et al. Rapid mobilization of murine and human hematopoietic stem and progenitor cells with AMD3100, a CXCR4 antagonist. J. Exp Med. 201, 1307-1318 (2005).

27. Azab, A. K. et al. CXCR4 inhibitor AMD3100 disrupts the interaction of multiple myeloma cells with the bone marrow microenvironment and enhances their sensitivity to therapy. Blood 113, 4341-4351 (2009).

28. Ceradini, D. J. et al. Progenitor cell trafficking is regulated by hypoxic gradients through HIF-1 induction of SDF-1. Nat. Med 10, 858-864 (2004).

29. Singh, S. R. et al. CCL2 release by airway smooth muscle is increased in asthma and promotes fibrocyte migration. Allergy 69, 1189-1197 (2014).

30. Phillips, R. J. et al. Circulating fibrocytes traffic to the lungs in response to CXCL12 and mediate fibrosis. J. Clin. Invest. 114, 438-446 (2004).

31. Jain, R. K. Normalization of tumor vasculature: an emerging concept in antiangiogenic therapy. Science 307, 58-62 (2005).
32. Mancuso, M. R. et al. Rapid vascular regrowth in tumors after reversal of VEGF inhibition. J. Clin. Invest. 116, 2610-2621 (2006).

33. Chung, A. S. et al. An interleukin-17-mediated paracrine network promotes tumor resistance to anti-angiogenic therapy. Nat. Med. 19, 1114-1123 (2013).

34. Bucala, R., Spiegel, L. A., Chesney, J., Hogan, M. \& Cerami, A. Circulating fibrocytes define a new leukocyte subpopulation that mediates tissue repair. Mol. Med. 1, 71-81 (1994).

35. Bellini, A. \& Mattoli, S. The role of the fibrocyte, a bone marrow-derived mesenchymal progenitor, in reactive and reparative fibroses. Lab. Invest. 87, 858-870 (2007).

36. Chesney, J., Metz, C., Stavitsky, A. B., Bacher, M. \& Bucala, R. Regulated production of type I collagen and inflammatory cytokines by peripheral blood fibrocytes. J. Immunol. 160, 419-425 (1998).

37. Gomperts, B. N. \& Strieter, R. M. Fibrocytes in lung disease. J. Leukoc. Biol. 82 449-456 (2007)

38. Rock, J. R. et al. Multiple stromal populations contribute to pulmonary fibrosis without evidence for epithelial to mesenchymal transition. Proc. Natl Acad. Sci. USA 108, E1475-E1483 (2011).

39. Sangaletti, S. et al. SPARC oppositely regulates inflammation and fibrosis in bleomycin-induced lung damage. Am. J. Pathol. 179, 3000-3010 (2011).

40. Aono, Y. et al. Surfactant protein D regulates effector cell function and fibrotic ung remodeling in response to bleomycin injuruy. Am. J. Respir. Crit. Care Med. 185, 525-536 (2012).

41. Yeager, M. E. et al. Circulating fibrocytes are increased in children and young adults with pulmonary hypertension. Eur. Respir. J. 39, 104-111 (2012).

42. Wang, C. H. et al. Increased circulating fibrocytes in asthma with chronic airflow obstruction.. Am. J. Respir. Crit. Care Med. 178, 583-591 (2008).

43. Medbury, H. J. et al. Monocytes contribute to the atherosclerotic cap by transformation into fibrocytes. Int. Angiol. 27, 114-123 (2008).

44. Mathai, S. K. et al. Circulating monocytes from systemic sclerosis patients with interstitial lung disease show an enhanced profibrotic phenotype. Lab. Invest. 90, 812-823 (2010)

45. Galligan, C. L. \& Fish, E. N. Circulating fibrocytes contribute to the pathogenesis of collagen antibody-induced arthritis. Arthritis Rheum. 64, 3583-3593 (2012).

46. Zhang, H. et al. Fibrocytes represent a novel MDSC subset circulating in patients with metastatic cancer. Blood 122, 1105-1113 (2013).

47. Willett, C. G. et al. Efficacy, safety, and biomarkers of neoadjuvant bevacizumab, radiation therapy, and fluorouracil in rectal cancer: a multidisciplinary phase II study. J. Clin. Oncol. 27, 3020-3026 (2009).

48. Dowlati, A. et al. Cell adhesion molecules, vascular endothelial growth factor, and basic fibroblast growth factor in patients with non-small cell lung cancer treated with chemotherapy with or without bevacizumab: an Eastern Cooperative Oncology Group study. Clin. Cancer Res. 14, 1407-1412 (2008).

49. Kopetz, S. et al. Phase II trial of infusional fluorouracil, irinotecan, and bevacizumab for metastatic colorectal cancer: efficacy and circulating angiogenic biomarkers associated with therapeutic resistance. J. Clin. Oncol. 28, 453-459 (2010).

50. Baar, J. et al. A vasculature-targeting regimen of preoperative docetaxel with or without bevacizumab for locally advanced breast cancer: impact on angiogenic biomarkers. Clin. Cancer Res. 15, 3583-3590 (2009).

51. Boige, V. et al. Efficacy, safety, and biomarkers of single-agent bevacizumab therapy in patients with advanced hepatocellular carcinoma. Oncologist 17, 1063-1072 (2012).

52. Loupakis, F. et al. Pharmacodynamic and pharmacogenetic angiogenesisrelated markers of first-line FOLFOXIRI plus bevacizumab schedule in metastatic colorectal cancer. Br. J. Cancer 104, 1262-1269 (2011).

53. Casanovas, O., Hicklin, D. J., Bergers, G. \& Hanahan, D. Drug resistance by evasion of antiangiogenic targeting of VEGF signaling in late-stage pancreatic islet tumors. Cancer Cell 8, 299-309 (2005).

54. Bergers, G., Song, S., Meyer-Morse, N., Bergsland, E. \& Hanahan, D. Benefits of targeting both pericytes and endothelial cells in the tumor vasculature with kinase inhibitors. J. Clin. Invest. 111, 1287-1295 (2003).

55. Batchelor, T. T. et al. AZD2171, a pan-VEGF receptor tyrosine kinase inhibitor normalizes tumor vasculature and alleviates edema in glioblastoma patients. Cancer Cell 11, 83-95 (2007)

56. Pollard, J. W. Tumour-educated macrophages promote tumour progression and metastasis. Nat. Rev. Cancer. 4, 71-78 (2004).

57. De Palma, M. et al. Tie2 identifies a hematopoietic lineage of proangiogenic monocytes required for tumor vessel formation and a mesenchymal population of pericyte progenitors. Cancer Cell 8, 211-226 (2005).

58. Hattori, K. et al. Placental growth factor reconstitutes hematopoiesis by recruiting VEGFR1 $(+)$ stem cells from bone-marrow microenvironment. Nat. Med 8, 841-849 (2002).

59. Kaplan, R. N. et al. VEGFR1-positive haematopoietic bone marrow progenitors initiate the pre-metastatic niche. Nature 438, 820-827 (2005). 
60. Bunt, S. K., Sinha, P., Clements, V. K., Leips, J. \& Ostrand-Rosenberg, S. Inflammation induces myeloid-derived suppressor cells that facilitate tumor progression. J. Immunol. 176, 284-290 (2006).

61. Yang, L. et al. Expansion of myeloid immune suppressor $\mathrm{Gr}+\mathrm{CD} 11 \mathrm{~b}+$ cells in tumor-bearing host directly promotes tumor angiogenesis. Cancer Cell 6, 409-421 (2004).

62. Shi, Y. et al. Deficiency of Kruppel-like factor KLF4 in myeloid-derived suppressor cells inhibits tumor pulmonary metastasis in mice accompanied by decreased fibrocytes. Oncogenesis 3, e129 (2014).

63. Nakataki, E. et al. Novel orthotopic implantation model of human malignant pleural mesothelioma (EHMES-10 cells) highly expressing vascular endothelial growth factor and its receptor. Cancer Sci. 97, 183-191 (2006).

64. Yano, S. et al. Novel metastasis model of human lung cancer in SCID mice depleted of NK cells. Int. J. Cancer 67, 211-217 (1996).

65. Yano, S. et al. Treatment for malignant pleural effusion of human lung adenocarcinoma by inhibition of vascular endothelial growth factor receptor tyrosine kinase phosphorylation. Clin. Cancer Res. 6, 957-965 (2000).

66. Weidner, N., Semple, J. P., Welch, W. R. \& Folkman, J. Tumor angiogenesis and metastasis-correlation in invasive breast carcinoma. N. Engl. J. Med. 324, 1-8 (1991).

67. Aono, Y. et al. Role of platelet-derived growth factor/platelet-derived growth factor receptor axis in the trafficking of circulating fibrocytes in pulmonary fibrosis. Am. J. Respir. Cell Mol. Biol. 51, 793-801 (2014).

68. Nishioka, Y., Nishimura, N., Suzuki, Y. \& Sone, S. Human monocyte-derived and CD83 $(+)$ blood dendritic cells enhance NK cell-mediated cytotoxicity. Eur. J. Immunol. 31, 2633-2641 (2001).

69. Moore, B. B. et al. CCR2-mediated recruitment of fibrocytes to the alveolar space after fibrotic injury. Am. J. Pathol. 166, 675-684 (2005).

\section{Acknowledgements}

We thank Drs I.J. Fidler (MD Anderson Cancer Center, TX, USA), Y. Sekido (Aichi Cancer Center Research Institute, Japan) and H. Hamada (Hiroshima University, Japan) for providing cell lines, and Dr M. Miyasaka (Osaka University, Japan) for providing TM- $\beta 1$. We also thank our colleagues at The University of Tokushima, especially Dr M. Shono, H. Umemoto and T. Oka for technical assistance with the immunohistochemistry and primary cell isolation, Dr H. Horikawa for the microarray analysis, and Dr Koji Yasutomo (Department of Immunology and Parasitology) and the members of the Nishioka lab for technical advice and fruitful discussions. This study was partly supported by a grant from Core Research and Evolutional Science and Technology,
Japan Science and Technology Corporation, and a grant from the Ministry of Health, Labour and Welfare of Japan awarded to the Study Group on Diffuse Pulmonary Disorders, Scientific Research/Research on intractable diseases, JSPS KAKENHI Grant Number 26461191, and the Practical Research Project for Rare Intractable Diseases from Japan Agency for Medical Research and Development, AMED.

\section{Author contributions}

H.G. and Y.N. designed, planned and coordinated the experiments and wrote the manuscript. A.M. wrote the manuscript and performed and analysed the experiments with A.S., V.T.T., Y.A., H.O., T.K. and S.T. S.K. and M.H. provided technical assistance. H.U. and K.I. assisted with the immunohistochemical studies. M.Y., H.K., H.T., M.G., H.Y. and S.S. provided the clinical specimens. S.Y. assisted with the study design. Y.N. supervised the project. All authors edited or commented on the manuscript.

\section{Additional information}

Accession codes: The microarray data were deposited in the NCBI Gene Expression Omnibus (GEO) under accession code GSE59476.

Supplementary Information accompanies this paper at http://www.nature.com/ naturecommunications

Competing financial interests: Y.N. has received research support from Chugai and Taiho Pharmaceuticals. The remaining authors declare no competing financial interests

Reprints and permission information is available online at http://npg.nature.com/ reprintsandpermissions/

How to cite this article: Mitsuhashi, A. et al. Fibrocyte-like cells mediate acquired resistance to anti-angiogenic therapy with bevacizumab. Nat. Commun. 6:8792 doi: $10.1038 /$ ncomms9792 (2015).

\section{(c) (i)}

This work is licensed under a Creative Commons Attribution 4.0 International License. The images or other third party material in this article are included in the article's Creative Commons license, unless indicated otherwise in the credit line; if the material is not included under the Creative Commons license, users will need to obtain permission from the license holder to reproduce the material. To view a copy of this license, visit http://creativecommons.org/licenses/by/4.0/ 